\title{
The genus Amiota (Diptera: Drosophilidae) from Hengduan Mountains, southwestern China
}

\author{
WenXia ZHANG ${ }^{1}$ and Hongwei CHEN $^{2 *}$ \\ ${ }^{1}$ College of Life Sciences, Peking University, 100871 Beijing, China \\ ${ }^{2}$ Department of Entomology, South China Agricultural University, Wushan 483, Tianhe, 510642 Guangzhou, China
}

\begin{abstract}
Keywords. Taxonomy, Diptera, Drosophilidae, Amiota, new species, Hengduan Mountains, China
\end{abstract}
\begin{abstract}
A total of 39 Amiota species are found from the southern portion of Hengduan Mountains, southwestern China, including 12 new species: A. gaoi sp. n., A. gracilenta sp. n., A. multispinata sp. n., A. yifengi sp. n., A. angustifolia sp. n., A. bacillia sp. n., A. biacuta sp. n., A. cultella sp. n., A. deltoidea sp. n., A. pianmensis sp. n., A. setosa sp. n. and A. bifoliolata sp. n. A key to all the studied species from Hengduan Mountains is provided.
\end{abstract}

\section{INTRODUCTION}

The Hengduan Mountains lie in the eastern Himalayan range and comprise a series of spectacular north-south trending ridges and valleys from eastern Qinghai-Xizang (Tibet) Plateau to Sichuan and Yunnan Provinces in southwestern China. In this region, the flora diversity is far greater than in any other region of comparable size in the temperate zone (Raven \& Axelrod, 1978; Wu, 1988); lower elevations (usually altitude of ca. 1000-2000 m) being characterized by subtropical forests. This region coincides in large part with the eastern Himalayan diversity "hot-spot" recognized by Meyers (1988) and Wilson (1992).

Since 1986, we researched on the fauna of drosophilids in the southern portion of the Hengduan Mountains (Deqin, Shangrila, Gongshan, Ninglang, Weixi, Lanping, Binchuan, Lushui, Kunming and Jingdong of Yunnan Province). Only in the genus Amiota, a total of 39 species have been found, nine of them (A. acuta, A. angulisternita, A. aquilotaurusata, A. dentata, A. furcata, A. onchopyga, A. subfurcata, A. trifurcata and A. stylopyga) having been reported from the eastern part of the Palaearctic Region, including North Japan (Honshu and Hokkaido by Wakahama \& Okada, 1958; Okada, 1960, 1968, 1971; Nishiharu, 1979; Takada et al., 1979), North to Northeast China (Jilin, Liaoning and Beijing by Toda et al., 1996; Chen \& Toda, 2001; Chen et al., 2004), Korea and Russia (Far East and East Siberia by Toda et al., 1996); the others are known only from South China. Endemic species-groups of the Oriental Region, i.e. the $A$. apodemata, A. sinuata and $A$. nagatai species-groups (Chen \& Toda, 1998a, b, 2001) are not present in the Hengduan Mountains. Therefore it seems that the southern portion of the Hengduan Mountains is an overlapping area of the Palaearctic and the Oriental Regions for the fauna of the genus Amiota, which is perhaps due to the influence of the Quaternary glacial period in the Cenozoic. In the Quaternary glacial period, some of the
Palaearctic drosophilids from the Northeast were forced to disperse to the Hengduan Mountains region. As the high mountains obstructed the advance of glacier, these areas were never affected by glaciation and became an ideal refuge for many animals. While in the interglacial period, some of the species migrated and inhabited the high mountains. The high mountains also acted as a natural barrier that prevented the genetic exchange among populations, and thus caused an intensification in speciation. These may be some of the reasons for this area to have evolved such an abundant diversity of species.

\section{MATERIAL AND METHODS}

The specimens used for observing and describing the morphology are deposited in part as dry specimens and in part preserved into $70 \%$ ethanol in the following institutions: College of Life Science, Peking University, Beijing, China (PKU); Kunming Institute of Zoology, Chinese Academy of Sciences, Kunming, China (KIZ); Department of Entomology, South China Agricultural University, Guangzhou, China (SCAU); Systematic Entomology, the Hokkaido University Museum, Hokkaido University, Sapporo, Japan (SEHU). New records are indicated by an asterisk $(*)$.

Morphological terminology and indices follow Chen \& Toda (2001) or Chen \& Aotsuka (2003).

\section{SYSTEMATIC ACCOUNT}

\section{Amiota Loew, 1862}

Amiota Loew, 1862: 229; Máca, 2003: 255. Type-species: Amiota leucostoma Loew, 1862.

Amiota (s. str.): Wheeler, 1952: 166; Okada, 1960: 89, 1968: 303, 1971: 82; Máca, 1980: 328, 2003: 255, 263; Máca \& Lin, 1993: 1; Chen \& Toda, 2001: 1517; Chen et al., 2005: 266.

Diagnosis. Face, postpronotal lobe and wing base each with distinctly milky white spots (absent in Amiota minor Malloch from the Nearctic; Máca, 2003); clypeus dark brown to black; aedeagal apodeme broad, dorso-ventrally

\footnotetext{
* Corresponding author; e-mail: hongweic@scau.edu.cn
} 
flattened; a few anterior sensilla of medial cibarials short, somewhat peg-like (Chen \& Toda, 2001).

For detailed description see Chen \& Toda (2001) and Chen et al. (2004, 2005).

\section{Amiota basdeni species-group}

Amiota (Amiota) basdeni species-group, Chen \& Toda, 2001: 1531; Chen et al., 2004: 61; Chen et al., 2005: 275.

Diagnosis. Surstylus with one to five aristate processes on mesal surface (Figs 1, 5, 10, 15); vertical lobe of gonopod with two sclerotized, basally fused M-shaped processes (Figs 6, 11).

Included species. A. angulisternita Chen \& Liu, 2004; A. aristata Chen \& Toda, 2001; A. basdeni Fonseca, 1965; A. brevipartita Chen \& Gao, 2005; A. clavata Okada, 1971; A. curvispina Chen \& Gao, 2005; A. curvistyla Okada, 1971; A. elongata Okada, 1971; A. flagellata Okada, 1971; A. huae Chen \& Gao, 2005; A. kitamurai Chen \& Liu, 2004; A. lipingae Chen \& Gao, 2005; A. longispinata Chen \& Gao, 2005; A. macai Chen \& Toda, 2001; A. onchopyga Nishiharu, 1979; A. palpifera Okada, 1971; . gaoi sp. n.; A. gracilenta sp. n.; $A$. multispinata sp. n.; A. yifengi sp. n.

\section{Amiota angulisternita Chen \& Liu, 2004}

Amiota (Amiota) angulisternita Chen \& Liu in Chen et al., 2004: 61

Specimens examined. Yunnan: 16호, Samage, Weixi, alt. $1900 \mathrm{~m}, 27-30 . v i i .2004,3 \hat{\delta}$ around human eyes, $13 \delta^{\star} \mathrm{ex}$ tree trunks, Y.-F. Li and H.-W. Chen.

Distribution. China (Liaoning, Taiwan, Yunnan*: Weixi).

\section{Amiota brevipartita Chen \& Gao, 2005}

Amiota (Amiota) brevipartita Chen \& Gao in Chen et al., 2005: 276.

Specimens examined. Yunnan: $5 \hat{\jmath}$, Samage, Weixi, alt. $1900 \mathrm{~m}, 27 .-30 . v i i .2004,1 \delta^{\star}$ around human eyes, 4ठ ex tree trunks, Y.-F. Li and H.-W. Chen.

Distribution. China (Yunnan: Ninglang, Weixi, Binchuan).

\section{Amiota curvispina Chen \& Gao, 2005}

Amiota (Amiota) curvispina Chen \& Gao in Chen et al., 2005: 277.

Specimens examined. Yunnan: $1 \hat{\delta}$, Samage, Weixi, alt. 1900 m, 27.vii.2004, ex tree trunks, H.-W. Chen.

Distribution. China (Yunnan: Weixi, Jingdong).

\section{Amiota lipingae Chen \& Gao, 2005}

Amiota (Amiota) lipingae Chen \& Gao in Chen et al., 2005: 278.

Specimens examined. Holotype: $\widehat{o}$, Yunnan: Lake Lugu, Ninglang, alt. 1800-2700 m, 23.vii.2001, L.P. He (KIZ).

Distribution. China (Yunnan: Ninglang).

\section{Amiota longispinata Chen \& Gao, 2005}

Amiota (Amiota) longispinata Chen \& Gao in Chen et al., 2005: 281.

Specimens examined. Yunnan: $15 \hat{\delta}$, Samage, Weixi, alt. $1900 \mathrm{~m}, 27 .-30 . v i i .2004,2 \hat{\delta}$ around human eyes, $13 \hat{\jmath}$ ex tree trunks, Y.-F. Li and H.-W. Chen.

Distribution. China (Yunnan: Weixi, Binchuan, Kunming).

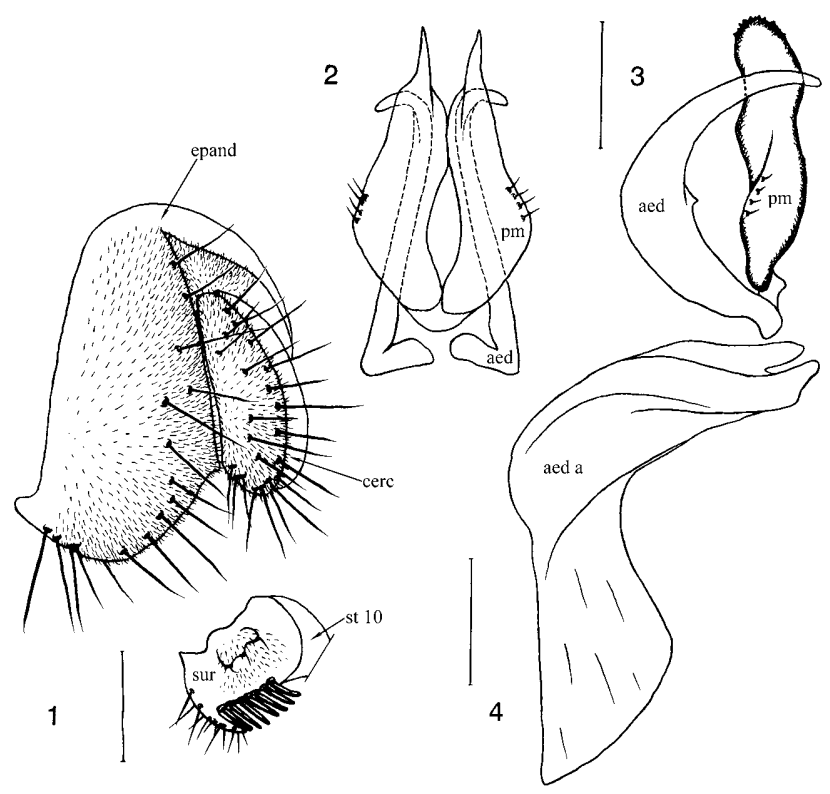

Figs 1-4. Amiota gaoi sp. n. $\mathbf{0}: 1$ - epandrium (epand), cercus (cerc) and surstylus (sur); 2 - parameres (pm) and basiphallus (aed) (ventral view); 3 - ditto (lateral view); 4 aedeagal apodeme (lateral view). Scale $0.1 \mathrm{~mm}$.

\section{Amiota macai Chen \& Toda, 2001}

Amiota (Amiota) macai Chen \& Toda, 2001: 1535.

Specimens examined. Yunnan: $3 \hat{\jmath}$, Lugu Lake, Ninglang, alt. 1800-2700 m, 22.-27.vii.2001, around human eyes, J.J. Gao.

Distribution. China (Hubei, Yunnan: Ninglang).

\section{Amiota onchopyga Nishiharu, 1979}

Amiota (Amiota) onchopyga Nishiharu, 1979: 39.

Specimens examined. Yunnan: $6 \hat{\jmath}$, Samage, Weixi, alt. $1900 \mathrm{~m}, 27 .-30 . v i i .2004,2 \widehat{t}$ around human eyes, $4{ }^{\dagger} \mathrm{ex}$ tree trunks, Y.-F. Li and H.-W. Chen.

Distribution. China (Fujian, Yunnan*: Weixi), Japan (Honshu).

\section{Amiota gaoi sp. n. (Figs 1-4)}

Diagnosis. Paramere thick, finely serrated on apical margin (Figs 2, 3); basiphallus basally thick, distally slender, medioventrally with one small, triangular projection (Figs 2, 3).

Description. Male: Hindleg femur and tibia lacking longer setae.

Abdomen: Lateral margin of sixth tergite slightly narrower than dorsomedian part.

Male terminalia: Epandrium constricted more than onehalf width mid-dorsally, with about 16 setae near posterior to ventral margins (Fig. 1). Surstylus pubescent medially, with three aristate processes basally fused to each other and forming palm-like lobe on mesal surface, one finger-like process at postero-ventral corner, about seven long prensisetae on distal margin (Fig. 1). Anterior portion of hypandrium slightly broadened at middle. Parameres basally slightly fused to each other, each submedially with four sensilla. Basiphallus divided into a pair of processes as long as paramere, curved, slightly 
sclerotized (Figs 2, 3); outer membrane high and erected basally.

Measurements. $\mathrm{BL}=3.03 \mathrm{~mm}$ in holotype (range in 5 paratypes: $2.72-3.12) ; \mathrm{ThL}=1.40 \mathrm{~mm}(1.30-1.56) ; \mathrm{WL}=2.90 \mathrm{~mm}$ $(2.82-3.00) ; \mathrm{WW}=1.20 \mathrm{~mm}(1.10-1.24)$. Indices: $a r b=5 / 4$ $(5 / 3-4)$, avd $=0.75(0.65-0.75)$, adf $=1.40(1.30-1.40)$, flw $=$ $1.70(1.60-1.75), \mathrm{FW} / \mathrm{HW}=0.36(0.34-0.40), \mathrm{ch} / \mathrm{o}=0.08$ $(0.07-0.09)$, prorb $=1.00(0.98-1.04)$, rcorb $=0.65(0.65-0.70)$, $\mathrm{vb}=0.35(0.30-0.35), \mathrm{dcl}=0.55(0.50-0.60)$, presctl $=0.65$ $(0.65-0.70)$, sctl $=1.10(1.00-1.10)$, sterno $=0.90(0.85-1.00)$, orbito $=1.50(1.50-1.54), \mathrm{dcp}=0.30(0.30)$, sctlp $=1.00$ (1.00-1.10), $\mathrm{C}=2.00(1.80-2.08), 4 \mathrm{c}=1.40(1.32-1.54), 4 \mathrm{v}=$ $2.14(2.11-2.46), 5 \mathrm{x}=1.42(1.36-1.46), \mathrm{ac}=3.90(3.82-4.88)$, $\mathrm{M}=0.61(0.62-0.65), \mathrm{C} 3 \mathrm{~F}=0.64(0.64-0.69)$.

Type material. Holotype: $\widehat{\delta}$, Yunnan: Samage, Weixi, alt. 1900 m, 27.-30.vii.2004, ex tree trunks, H.W. Chen (SCAU).

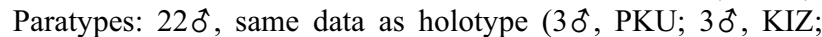
$13 \hat{\sigma}$, SCAU; $3 \hat{0}$, SEHU); Yunnan: $1 \hat{\sigma}$, Benzilan, Deqin, alt. $2700 \mathrm{~m}, 31 . v i i .2004$, ex tree trunks, H.-W. Chen (SCAU).

Etymology. Patronym, in honor of Dr J.-J. Gao (Yunnan University, China).

Distribution. China (Yunnan: Deqin, Weixi).

Remarks. This species is related to $A$. macai in the shape of basiphallus, but clearly distinguishable from the latter by the parameres (in A. macai: paramere lacking fine serrations on apical margin, narrowed from distal one-third in lateral view).

\section{Amiota gracilenta sp. n. (Figs 5-9)}

Diagnosis. Paramere and basiphallus slender, sclerotized, pointed apically (Figs 7, 8).

Description. Male: Hindleg femur and tibia lacking longer setae.

Abdomen: Lateral margin of sixth tergite slightly narrower than dorsomedian part.

Male terminalia: Epandrium slightly constricted middorsally, with about 15 setae near posterior to ventral margins (Fig. 5). Surstylus pubescent medially, with three to four aristate processes basally fused to each other and forming palm-like lobe on mesal surface, one finger-like process at postero-ventral corner, about nine long prensisetae on distal margin (Fig. 5). Anterior portion of hypandrium thin at middle (Fig. 6). Paramere submedially with five to six sensilla (Figs 7, 8). Basiphallus divided into a pair of processes as long as paramere, slightly sclerotized (Figs 7,8); outer membrane high and erected basally.

Measurements. $\mathrm{BL}=2.49 \mathrm{~mm}$ in holotype (range in 5 paratypes: $2.05-2.80), \mathrm{ThL}=1.14 \mathrm{~mm}(0.95-1.30), \mathrm{WL}=2.21 \mathrm{~mm}$ $(1.80-2.45), \mathrm{WW}=1.01 \mathrm{~mm}(0.85-1.13)$. Indices: $a r b=4 / 3$ $(4-5 / 3-4)$, avd $=0.75(0.65-0.75)$, adf $=1.40(1.35-1.50)$, flw $=$ $1.60(1.50-1.65), \mathrm{FW} / \mathrm{HW}=0.43(0.39-0.47), \mathrm{ch} / \mathrm{o}=0.12$ $(0.10-0.14)$, prorb $=0.96(0.77-1.11)$, rcorb $=0.74(0.63-0.89)$, $\mathrm{dcl}=0.57(0.52-0.62)$, presctl $=0.57(0.50-0.67)$, sctl $=1.19$ $(1.05-1.32)$, sterno $=0.79(0.63-0.87)$, orbito $=1.78$ (1.67-2.16), dcp $=0.27(0.26-0.29)$, sctlp $=1.11(1.00-1.17), \mathrm{C}$ $=1.71(1.56-1.96), 4 \mathrm{c}=1.68(1.56-1.90), 4 \mathrm{v}=2.70$ (2.47-3.03), $5 \mathrm{x}=1.58(1.28-1.86), \mathrm{ac}=4.00(3.71-4.50), \mathrm{M}=$ $0.71(0.61-0.87), \mathrm{C} 3 \mathrm{~F}=0.67(0.57-0.73)$.

Type material. Holotype: $\hat{\delta}$, Yunnan, Pengdang, Gongshan, alt. $1475 \mathrm{~m}, 23 . v i .1993$, around human eyes, W.-X. Zhang (KIZ). Paratypes: $3 \hat{0}$, same data as holotype (KIZ); $3 \hat{0}$, same

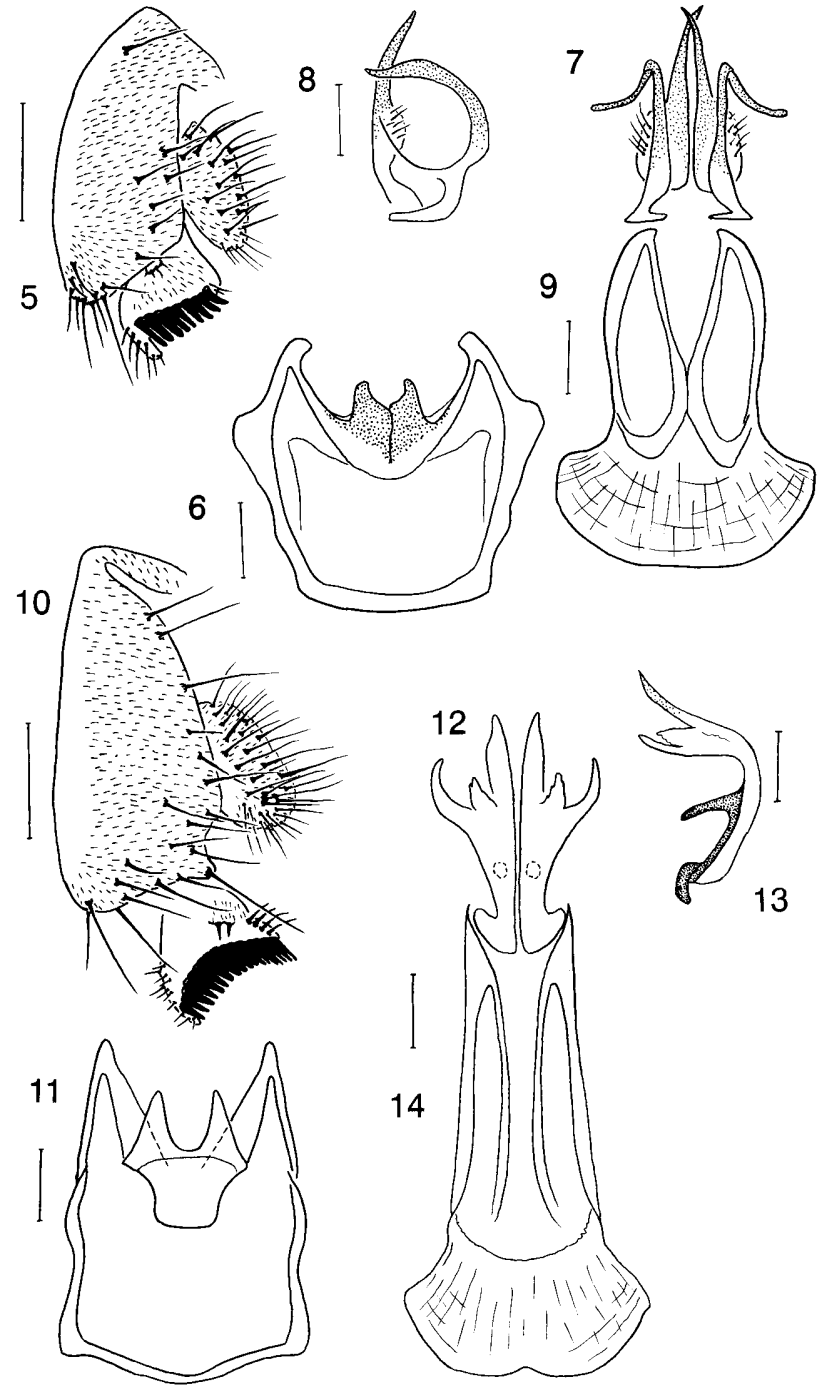

Figs 5-14. 5-9: Amiota gracilenta sp. n. đ: : 5 - epandrium, cercus and surstylus; 6 - hypandrium and gonopod (ventral view); 7 - parameres and basiphallus (ventral view); 8 - ditto (lateral view); 9 - aedeagal apodeme (ventral view). 10-14: Amiota multispinata sp. n. $\widehat{0}$ : 10 - epandrium, cercus and surstylus; 11 - hypandrium and gonopod (ventral view); 12 parameres (ventral view); 13 - paramere and basiphallus (lateral view); 14 - aedeagal apodeme (ventral view). Scale $0.1 \mathrm{~mm}$.

data as holotype except for 24.vi.1993 (PKU); Yunnan: $2 \sigma^{\star}$, Laowo, Lanping, alt. 1450 m, 29.vi.1993, around human eyes, L.-X. Han (PKU); 15 §, Mt. Jizu, Binchuan, 17.-19.viii.2000, $5 \hat{\sigma}$ around human eyes, $10 \hat{\jmath}$ ex tree trunks, H.-W. Chen $(5 \hat{\sigma}$,

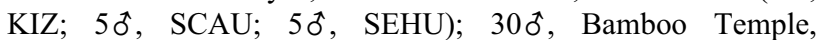

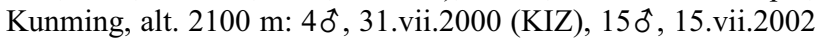
(KIZ), $11 \hat{\delta}, 20 .-21$.vii 2004 (SCAU), $13 \hat{\jmath}$ around human eyes, $17 \delta$ ex tree trunks, J.-J. Gao and H.-W. Chen; 50, Lugu Lake, Ninglang, alt. 1800-2470 m, 22.-27.vii.2001, around human eyes, J.-J. Gao (KIZ); 20ิ, Samage, Weixi, alt. 1900 m, 27.-30.vii.2004, around human eyes, Y.-F. Li (SCAU).

Etymology. From the Latin word gracilis meaning thin, referring to the slender paramere.

Distribution. China (Yunnan: Ninglang, Weixi, Lanping, Binchuan, Kunming). 
Remarks. This species is related to A. aristata in the shape of basiphallus, but clearly distinguishable from the latter by the paramere (in A. aristata: paramere three times broader than basiphallus medially in lateral view).

Amiota multispinata sp. n. (Figs 10-14)

Diagnosis. Paramere weakly sclerotized, lacking sensilla, distally tripartite (Figs 12, 13); basiphallus slender, slightly sclerotized, medially entirely fused to base of paramere (Fig. 13).

Description. Male: Hindleg femur and tibia lacking longer setae.

Abdomen: Lateral margin of sixth tergite slightly narrower than dorsomedian part.

Male terminalia: Epandrium constricted more than onehalf width mid-dorsally, with about 20 setae near posterior to ventral margins (Fig. 10). Surstylus pubescent medially, with one to two aristate processes basally not fused to each other on mesal surface, one finger-like process at postero-ventral corner, about 18 long prensisetae on distal margin (Fig. 10). Anterior portion of hypandrium thin at middle (Fig. 11). Aedeagal outer membrane high and erected basally.

Measurements. $\mathrm{BL}=3.18 \mathrm{~mm}$ in holotype (range in 2 paratypes: $3.00-3.50), \mathrm{ThL}=1.51 \mathrm{~mm}(1.45-1.60), \mathrm{WL}=2.69 \mathrm{~mm}$ $(2.50-2.80), \mathrm{WW}=1.28 \mathrm{~mm}(1.20-1.30)$. Indices: arb $=$ $4-5 / 3-4 \quad(4-5 / 3-4)$, avd $=0.65(0.65-0.75)$, adf $=1.30$ $(1.35-1.45), \quad$ flw $=1.50 \quad(1.50-1.60), \quad \mathrm{FW} / \mathrm{HW}=0.43$ $(0.42-0.46), \mathrm{ch} / \mathrm{o}=0.13(0.11-0.14)$, prorb $=1.05(1.00-1.09)$, rcorb $=0.80(0.60-0.93), \mathrm{dcl}=0.50(0.44-0.54)$, presctl $=0.56$ $(0.48-0.64)$, sctl $=1.06(1.04-1.08)$, sterno $=0.90(0.82-0.95)$, orbito $=1.55(1.50-1.71), \mathrm{dcp}=0.23(0.21-0.24), \mathrm{sctlp}=1.27$ (1.13-1.57), $\mathrm{C}=1.49(1.41-1.64), 4 \mathrm{c}=1.86(1.76-1.95), 4 \mathrm{v}=$ 2.27 (2.59-2.90), $5 \mathrm{x}=1.52(1.21-1.78), \mathrm{ac}=4.57(4.35-4.88)$, $\mathrm{M}=0.71(0.62-0.80), \mathrm{C} 3 \mathrm{~F}=0.80(0.77-0.82)$.

Type material. Holotype: $\widehat{\delta}$, Yunnan: Pengdang, Gongshan, alt. $1475 \mathrm{~m}, 23 . v i .1993$, around human eyes, W.-X. Zhang (KIZ). Paratypes: $2 \hat{0}$, same data as holotype (PKU); $1 \hat{\widehat{t}}$, same data as holotype except for alt. $1520 \mathrm{~m}, 24 . v i .1993$ (SCAU).

Etymology. A combination of the Latin words: multus and spina, referring to the surstylus with multiple prensisetae.

Distribution. China (Yunnan: Gongshan).

Remarks. This species is related to A. flagellata in the paramere fused to the basiphallus basally, but differs from the latter by the shape of the paramere and basiphallus (in A. flagellata: paramere and basiphallus heavily sclerotized, pointed apically).

\section{Amiota yifengi sp. n. (Figs 15-18)}

Diagnosis. Basiphallus basally slightly curved, distally straight in lateral and ventral view (Fig. 17).

Description. Male: Hindleg femur and tibia lacking longer setae.

Abdomen: Lateral margin of sixth tergite slightly narrower than dorsomedian part.

Male terminalia: Epandrium constricted more than onehalf width mid-dorsally, with about 12 setae near posterior to ventral margins (Fig. 15). Surstylus pubescent basally, with one small aristate process on mesal surface, one finger-like process at postero-ventral corner, and about 13 long prensisetae on distal margin (Fig. 15).
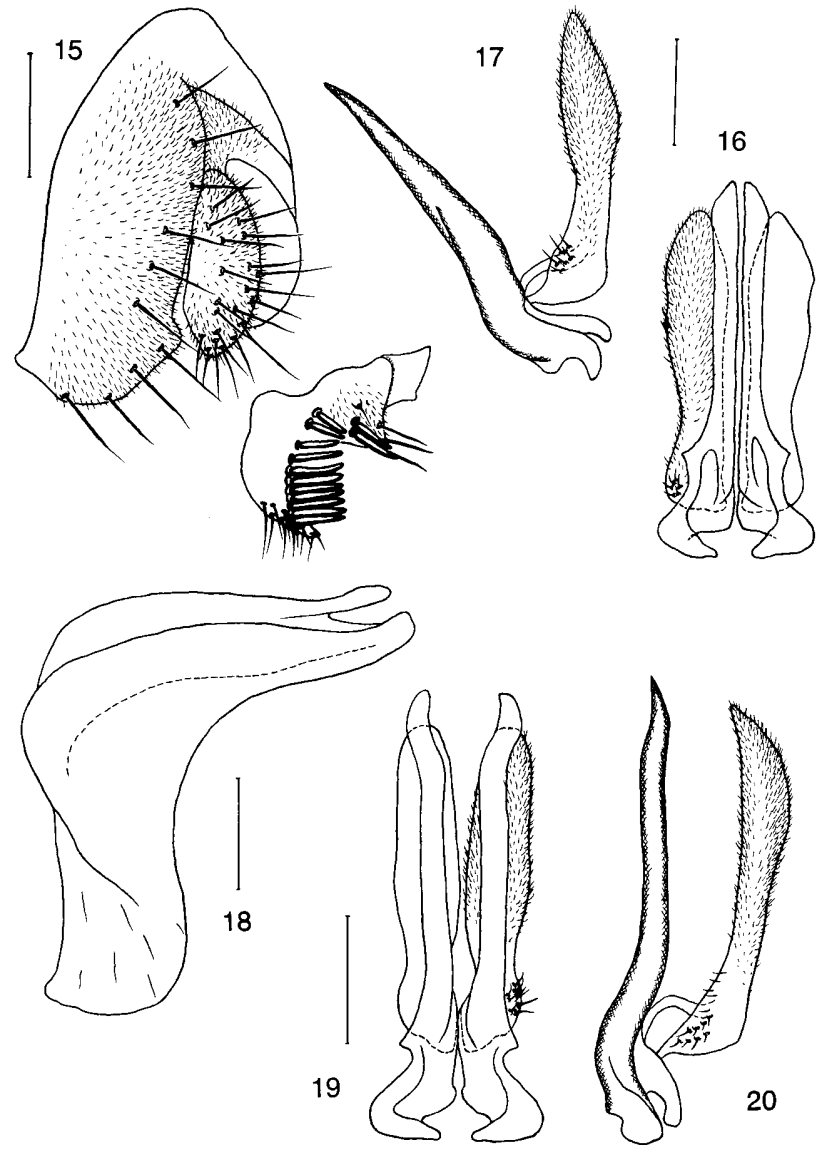

Figs 15-20. 15-18: Amiota yifengi sp. n. đે: 15 - epandrium, cercus and surstylus; 16 - parameres and basiphallus (ventral view); 17 - ditto (lateral view); 18 - aedeagal apodeme (lateral view). 19-20: Amiota palpifera Okada ot: 19 - parameres and basiphallus (ventral view); 20 - ditto (lateral view). Scale 0.1 $\mathrm{mm}$.

Anterior portion of hypandrium slightly broadened at middle. Paramere entirely densely pubescent, and slightly sclerotized, with about six sensilla basally (Figs 16, 17). Basiphallus divided into a pair of processes longer than paramere, strongly sclerotized (Figs 16, 17); outer membrane high and erected basally.

Measurements. $\mathrm{BL}=3.07 \mathrm{~mm}$ in holotype (range in $7 \mathrm{~B}$ paratypes: $2.86-3.26)$; $\mathrm{ThL}=1.45 \mathrm{~mm}(1.30-1.50)$; $\mathrm{WL}=2.86$ $\mathrm{mm}(2.73-2.93) ; \mathrm{WW}=1.15 \mathrm{~mm}(1.10-1.25)$. Indices: $\operatorname{arb}=$ $4-5 / 3-4(4-5 / 3-4)$, avd $=0.70(0.65-0.75)$, adf $=1.40$ $(1.35-1.55), \mathrm{flw}=1.60(1.60-1.70), \mathrm{FW} / \mathrm{HW}=0.40(0.40), \mathrm{ch} / \mathrm{o}$ $=0.08(0.08-0.10)$, prorb $=1.00(1.00-1.10)$, rcorb $=0.70$ $(0.60-0.70), \mathrm{dcl}=0.60(0.55-0.65)$, presctl $=0.65(0.65-0.70)$, sctl $=1.10(1.00-1.10)$, sterno $=0.90(0.86-0.95)$, orbito $=1.55$ $(1.45-1.65), \mathrm{dcp}=0.25(0.25)$, sctlp $=1.15(1.10-1.20), \mathrm{C}=$ $1.47(1.40-1.60), 4 \mathrm{c}=1.86(1.62-1.96), 4 \mathrm{v}=2.37(2.19-2.50)$, $5 \mathrm{x}=1.42(1.31-1.68), \mathrm{ac}=4.43(4.35-4.88), \mathrm{M}=0.73$ $(0.64-0.80), \mathrm{C} 3 \mathrm{~F}=0.80(0.70-0.86)$.

Type material. Holotype: ô, Yunnan: Samage, Weixi, alt. 1900 m, 27.-30.vii.2004, ex tree trunks, H.-W. Chen (SCAU). Paratypes: $7 \hat{\delta}$, same data as holotype $(2 \hat{0}, \mathrm{KIZ}$; 3B, SCAU; 20ิ, SEHU); Yunnan: $3 \hat{0}$, Pengdang, Gongshan, 23.vi.1993, around human eyes, W.-X. Zhang (PKU).

Etymology. Patronym, in honor of Mr Y.-F. Li (SCAU).

Distribution. Yunnan (Weixi, Gongshan). 
Remarks. This species was indistinguishable from $A$. palpifera by Chen et al. (2005) based on only one specimen examined. In the present study, we checked all relevant specimens collected from Yunnan Province and found them different from $A$. palpifera in the shape of basiphallus (in $A$. palpifera: basiphallus apically curved in both lateral and ventral view as shown in Figs 19, 20, based on $3 \hat{\sigma}$ specimens from Yamanashi, Honshu, Japan).

\section{Amiota taurusata species-group}

Amiota (Amiota) taurusata species-group, Chen \& Toda, 2001: 1536; Chen et al., 2004: 63; Chen et al., 2005: 282.

Diagnosis. Male: Hindleg femur with small, lobe-like flap baso-ventrally; fourth tergite laterally broadened and protruded more than others.

Included species. A. aquilotaurusata Takada, Beppu \& Toda, 1979; A. asymmetrica Chen \& Takamori, 2005; $A$. femorata Chen \& Takamori, 2005; A. sacculipes Máca \& Lin, 1993; A. taurusata Takada, Beppu \& Toda, 1979; A. vulnerabla Chen \& Zhang, 2004; A. yixiangensis Chen \& Takamori, 2005.

\section{Amiota aquilotaurusata Takada, Beppu \& Toda, 1979}

Amiota (Amiota) aquilotaurusata Takada, Beppu \& Toda, 1979: 110.

Specimens examined. Yunnan: $1 \hat{\jmath}$, Benzilan, Deqin, alt. 2700 m, 31.vii.2004, ex tree trunks, H.-W. Chen.

Distribution. Russia (Ussuri Region), China (Heilongjiang, Liaoning, Beijing, Yunnan*: Deqin), Japan (Hokkaido).

\section{Amiota asymmetrica Chen \& Takamori, 2005}

Amiota (Amiota) asymmetrica Chen \& Takamori in Chen et al., 2005: 283 .

Specimens examined. Yunnan: 1 $\widehat{0}$, Samage, Weixi, alt. 1900 m., 27.vii.2004, ex tree trunks, H.-W. Chen.

Distribution. China (Yunnan: Binchuan, Ninglang, Weixi).

\section{Amiota femorata Chen \& Takamori, 2005}

Amiota (Amiota) femorata Chen \& Takamori in Chen et al., 2005: 284 .

Specimens examined. Yunnan: $4 \hat{\jmath}$, Samage, Weixi, alt. 1900 m, 27.-30.vii.2004, ex tree trunks, H.-W. Chen.

Distribution. China (Hunan, Yunnan: Binchuan, Weixi).

\section{Amiota alboguttata species-group}

Amiota (Amiota) alboguttata species-group, Chen \& Toda, 2001: 1537; Chen et al., 2004: 64; Chen et al., 2005: 287.

Diagnosis. Male: Hindleg tibia ventrally with a row of long setae; hindleg second to fifth tarsomeres broadened: second shorter than one-half of its width.

Included species. A. ailaoshanensis Chen \& Watabe, 2005; A. albilabris (Roth, 1860); A. alboguttata (Wahlberg, 1838); A. arcuata Chen \& Watabe, 2005; A. bifurcata Chen, 2004; A. communis Chen \& Steyskal, 2004; A. cuii Chen \& Toda, 2001; A . dehiscentia Chen \& Watabe, 2005; A. delta Takada, Beppu \& Toda, 1979; A. dispina Okada, 1960; A. eos Sidorenko, 1989; A. falcilis Takada, Beppu \& Toda, 1979; A. forficula Takada, Beppu \& Toda, 1979; A. jizushanensis Chen \& Watabe, 2005; A. lanceolata Okada, 1971; A. latitabula Chen \& Watabe,
2005; A. luguhuensis Chen \& Watabe, 2005; A. nozawai Chen \& Watabe, 2005; A. nuerhachii Chen \& Toda, 2001; A. paraspinata Chen \& Watabe, 2005; A. shangrila Chen \& Watabe, 2005; A. sigma Okada, 1971; A. spinata Chen \& Toda, 2001; A. subtusradiata Duda, 1934; $A$. todai Sidorenko, 1989; A. trifurcata Okada, 1968; A. watabei Chen \& Toda, 2001; A. angustifolia sp. n., A. bacillia sp. n.; A. biacuta sp. n.; A. cultella sp. n.; A. deltoidea sp. n.; A. pianmensis sp. n.; A. setosa sp. n.

\section{Amiota ailaoshanensis Chen \& Watabe, 2005}

Amiota (Amiota) ailaoshanensis Chen \& Watabe in Chen et al., 2005: 287.

Specimens examined. Holotype $\widehat{\delta}$, Yunnan: Mt. Ailao, Jingdong, alt. 1700-2450 m, 22.vi.2001, H. Watabe (KIZ).

Distribution. China (Yunnan: Jingdong).

Amiota arcuata Chen \& Watabe, 2005

Amiota (Amiota) arcuata Chen \& Watabe in Chen et al., 2005: 289.

Specimens examined. Paratype $1 \hat{\delta}$, Yunnan: Lake Lugu, Ninglang, alt. 1800-2700 m, 23.vii.2001, J.J. Gao (SCAU).

Distribution. China (Yunnan: Ninglang).

Amiota dehiscentia Chen \& Watabe, 2005

Amiota (Amiota) dehiscentia Chen \& Watabe in Chen et al., 2005: 290

Specimens examined. Holotype $\widehat{\delta}$, Yunnan: Mt. Jizu, Binchuan, alt. 2150 m, 18.viii.2000, H.W. Chen (KIZ).

Distribution. China (Yunnan: Binchuan).

Amiota jizushanensis Chen \& Watabe, 2005

Amiota (Amiota) jizushanensis Chen \& Watabe in Chen et al., 2005: 291.

Specimens examined. Holotype $\hat{\jmath}$, Yunnan: Mt. Jizu, Binchuan, alt. $2150 \mathrm{~m}$, 18.viii.2000, H.W. Chen (KIZ).

Distribution. China (Yunnan: Binchuan).

Amiota latitabula Chen \& Watabe, 2005

Amiota (Amiota) latitabula Chen \& Watabe in Chen et al., 2005: 292.

Specimens examined. Yunnan: $5 \hat{\sigma}$, Samage, Weixi, alt. $1900 \mathrm{~m}, 27 .-30 . v i i .2004,1 \delta$ around human eyes, $4 \hat{\sigma}$ ex tree trunks, Y.-F. Li and H.-W. Chen (SCAU).

Distribution. China (Yunnan: Kunming, Binchuan, Weixi).

Amiota luguhuensis Chen \& Watabe, 2005

Amiota (Amiota) luguhuensis Chen \& Watabe in Chen et al., 2005: 294.

Specimens examined. Holotype $\hat{\sigma}$, Yunnan: Lake Lugu, Ninglang, alt. 1800-2700 m, 25.vii.2001, L.-P. He (KIZ). Distribution. China (Yunnan: Ninglang).

Amiota nozawai Chen \& Watabe, 2005

Amiota (Amiota) nozawai Chen \& Watabe in Chen et al., 2005: 296.

Specimens examined. Yunnan: $6 \hat{0}$, Samage, Weixi, alt. $1900 \mathrm{~m}, 27 .-30 . v i i .2004,3 \delta$ around human eyes, $3 \delta$ ex tree trunks, Y.-F. Li and H.-W. Chen (SCAU).

Distribution. China (Hunan, Yunnan*: Weixi). 


\section{Amiota paraspinata Chen \& Watabe, 2005}

Amiota (Amiota) paraspinata Chen \& Watabe in Chen et al., 2005: 297.

Specimens examined. Yunnan: $3 \hat{\jmath}$, Samage, Weixi, alt. 1900 m, 27.-30.vii.2004, Y.-F. Li and H.-W. Chen (SCAU).

Distribution. China (Yunnan: Kunming, Binchuan, Weixi).

\section{Amiota shangrila Chen \& Watabe, 2005}

Amiota (Amiota) shangrila Chen \& Watabe in Chen et al., 2005. 298.

Specimens examined. Yunnan: $1 \hat{\sigma}$, Samage, Weixi, alt. 1900 m, 27.vii.2004, ex tree trunks, H.-W. Chen (SCAU).

Distribution. China (Yunnan: Ninglang, Weixi).

\section{Amiota trifurcata Okada, 1968}

Amiota (Amiota) trifurcata Okada, 1968: 308.

Specimens examined. Yunnan: $3 \hat{\sigma}$, Samage, Weixi, alt. 1900 m, 27.-29.vii.2004, ex tree trunks, H.-W. Chen (SCAU).

Distribution. Russia (Ussuri Region), China (Yunnan*: Weixi), Japan (Hokkaido, Honshu).

Amiota angustifolia sp. n. (Figs 21-25)

Diagnosis. Vertical lobe of gonopod heavily sclerotized distally with one acute process mid-apically, round apicolaterally (Fig. 23).

Description. Male: Thorax and abdomen nearly entirely black.

Hindleg femur with six long setae posteroventrally; hindleg tibia with five setae ventrally.

Abdomen: Sixth tergite dorsally broadened, laterally tapering, reaching lateral margin of fifth tergite.

Male terminalia: Epandrium nearly separated middorsally, with about 18 setae near posterior to ventral margins (Fig. 21). Surstylus lacking pubescence, not expanded at posteroventral corner, with six prensisetae on distal margin (Fig. 22). Tenth sternite bilobed, but not divided medially, laterally narrowly fused to surstyli (Fig. 22). Anterior portion of hypandrium entirely thin (Fig. 23). Paramere lobe-shaped, with six to seven sensilla medially (Fig. 24). Basiphallus divided into a pair of simple rods, each fused to base of paramere (Fig. 24).

Measurements. $\mathrm{BL}=2.87 \mathrm{~mm}$ in holotype; $\mathrm{ThL}=1.27 \mathrm{~mm}$; $\mathrm{WL}=2.52 \mathrm{~mm} ; \mathrm{WW}=1.16 \mathrm{~mm}$. Indices: $\mathrm{arb}=5 / 4$, avd $=0.57$, adf $=1.17, \mathrm{flw}=1.88, \mathrm{FW} / \mathrm{HW}=0.40, \mathrm{ch} / \mathrm{o}=0.10$, prorb $=$ $0.98, \mathrm{rcorb}=0.72, \mathrm{vb}=0.35, \mathrm{dcl}=0.65$, presctl $=0.55, \mathrm{sctl}=$ 1.23 , sterno $=0.89$, orbito $=1.52, \mathrm{dcp}=0.31, \mathrm{sctlp}=1.16, \mathrm{C}=$ $2.25,4 \mathrm{c}=1.41,4 \mathrm{v}=2.71,5 \mathrm{x}=1.19, \mathrm{ac}=3.91, \mathrm{M}=0.64, \mathrm{C} 3 \mathrm{~F}$ $=0.68$.

Type material. Holotype: $\hat{\sigma}$, Yunnan: Pianma, Lushui, alt. $1500 \mathrm{~m}, 15$.viii.2000, around human eyes, H.-W. Chen (SCAU).

Etymology. A combination of the Latin words: angustus and folium meaning narrow leaf, referring to the paramere.

Distribution. China (Yunnan: Lushui).

Remarks. This species closely resembles $A$. shangrila in many characters of male terminalia, but it can be separated from the latter by the gonopod (in A. shangrila: vertical lobe of gonopod clearly broader than its height, acute apicolaterally).

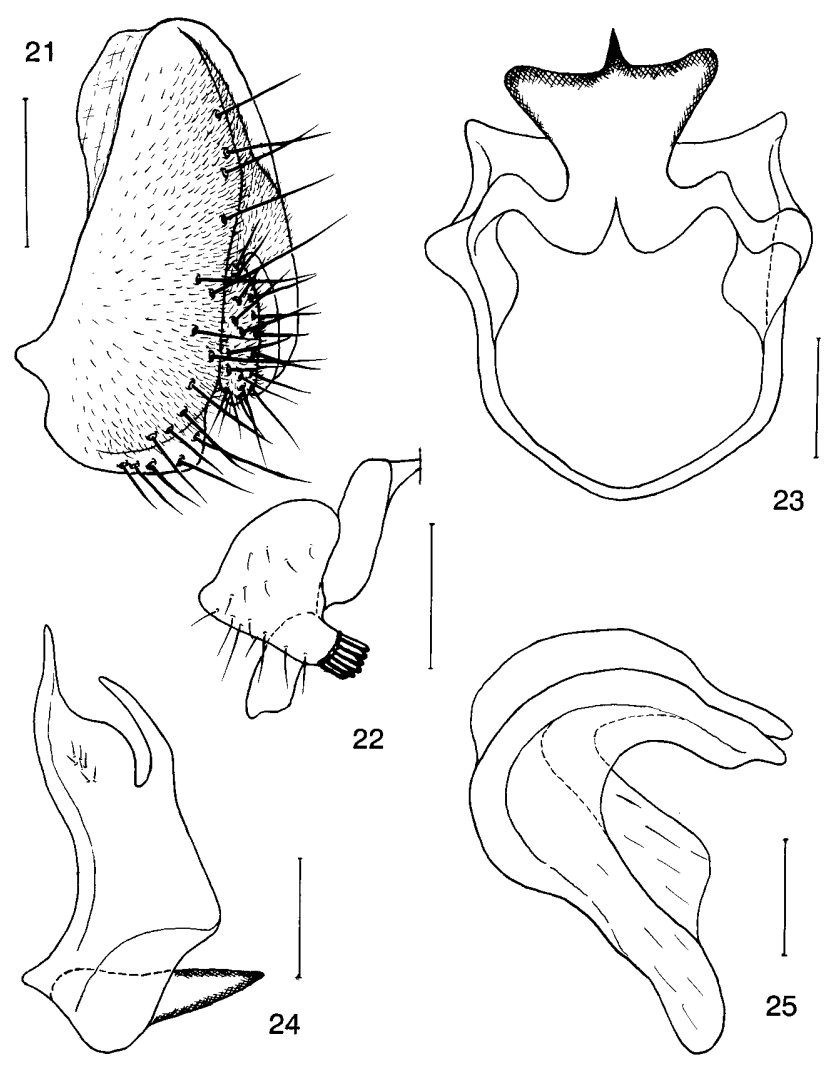

Figs 21-25. Amiota angustifolia sp. n. đ: 21 - Epandrium and cercus; 22 - surstylus and tenth sternite (ventral view); 23 hypandrium and gonopod (ventral view); 24 - paramere and basiphallus (ventral view); 25 - aedeagal apodeme (ventral view). Scale $0.1 \mathrm{~mm}$.

\section{Amiota bacillia sp. n. (Figs 26-30)}

Diagnosis. Vertical lobe of gonopod strongly sclerotized, distally protruded, nearly long rod-shaped, with one acute projection posteromedially (Figs 27, 28).

Description. Male: Hindleg femur with five long setae posteroventrally; hindleg tibia with seven setae ventrally.

Abdomen: Sixth tergite dorsally broadened, laterally tapering, reaching lateral margin of fifth tergite.

Male terminalia: Epandrium completely divided middorsally, with 11-12 setae near posterior to ventral margins (Fig. 26). Surstylus lacking pubescence, not expanded at posteroventral corner, with five prensisetae on distal margin (Fig. 26). Tenth sternite bilobed, but not divided medially, laterally narrowly fused to surstyli (Fig. 26). Anterior portion of hypandrium entirely thin (Fig. 27). Paramere lobe-shaped, with six to seven sensilla medially (Figs 29, 30). Basiphallus divided into a pair of simple rods, each fused to base of paramere (Figs 29, 30).

Measurements. $\mathrm{BL}=3.25 \mathrm{~mm}$ in holotype (range in 1 paratype: 3.50$), \mathrm{ThL}=1.45 \mathrm{~mm}(1.50), \mathrm{WL}=2.90 \mathrm{~mm}(3.00), \mathrm{WW}$ $=1.23 \mathrm{~mm}(1.33)$. Indices: $\mathrm{arb}=4 / 4(4 / 4)$, avd $=0.55(0.60)$, adf $=1.10(1.10)$, flw $=1.70(1.65), \mathrm{FW} / \mathrm{HW}=0.43(0.40), \mathrm{ch} / \mathrm{o}$ $=0.09(0.08)$, prorb $=1.08(1.00)$, rcorb $=0.75(0.70), \mathrm{dcl}=$ $0.48(0.55)$, prsctl $=0.56(0.65)$, sctl $=0.85(0.95)$, sterno $=0.88$ $(0.90)$, orbito $=2.00(2.00)$, dcp $=0.29(0.30)$, sctlp $=1.13$ (1.10), $\mathrm{C}=2.06(2.43), 4 \mathrm{c}=1.52(1.58), 4 \mathrm{v}=2.71(3.00), 5 \mathrm{x}=$ 

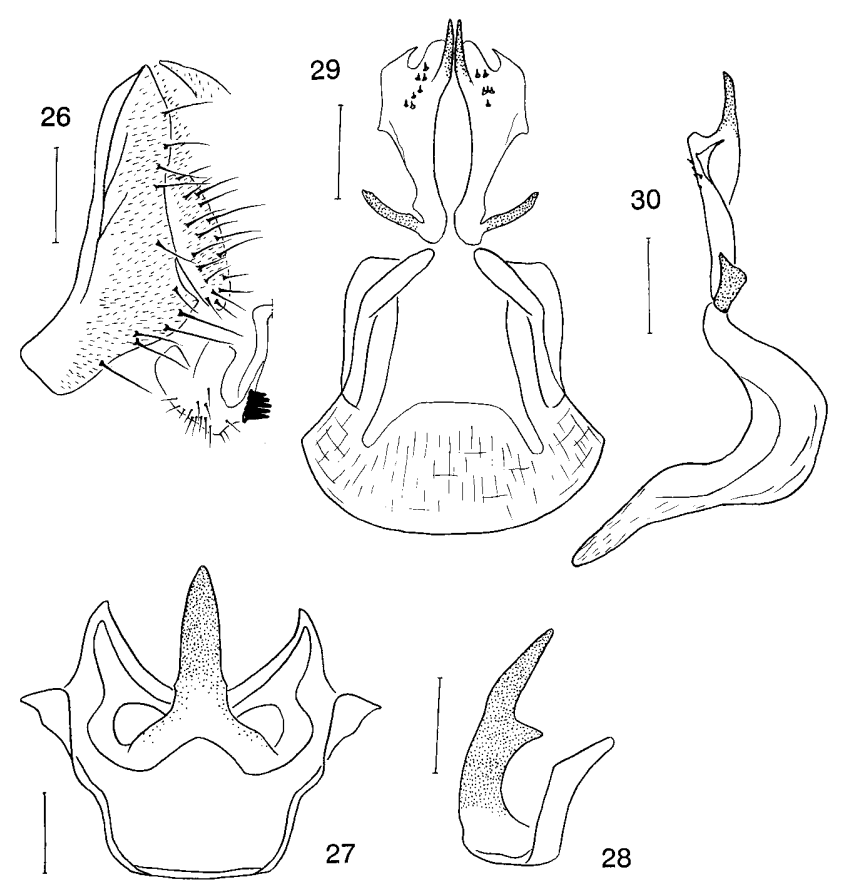

Figs 26-30. Amiota bacillia sp. n. đ: 26 - epandrium, cercus, surstylus and tenth sternite; 27 - hypandrium and gonopod (ventral view); 28 - gonopod (lateral view); 29 - parameres, basiphallus and aedeagal apodeme (ventral view); 30 - ditto (lateral view). Scale $0.1 \mathrm{~mm}$.

$1.44(1.90), \mathrm{ac}=4.92(4.29), \mathrm{M}=0.62(1.00), \mathrm{C} 3 \mathrm{~F}=0.78$ (0.79).

Type material. Holotype: $\hat{\jmath}$, Yunnan: Pengdang, Gongshan, alt. $1520 \mathrm{~m}, 24 . v i .1993$, around human eyes, W.-X. Zhang (KIZ). Paratype: 10, Yunnan: Pianma, Lushui, alt. $1500 \mathrm{~m}$, 13.viii.2000, around human eyes, H.-W. Chen (SCAU).

Etymology. From the Latin word: bacillus meaning small rod, referring to the rod-like vertical lobe of gonopod.

Distribution. China (Yunnan: Gongshan, Lushui).

Remarks. This species is related to A. ailaoshanensis, but it can be clearly distinguishable from the latter by the vertical lobe of gonopod (in A. ailaoshanensis: vertical lobe of gonopod basally broadened).

Amiota biacuta sp. n. (Figs 31-34)

Diagnosis. Vertical lobe of gonopod heavily sclerotized, with two long, pointed processes apically (Fig. 32).

Description. Male: Hindleg femur with seven to eight long setae posteroventrally; hindleg tibia with seven setae ventrally.

Abdomen: Sixth tergite dorsally broadened, laterally tapering, reaching lateral margin of fifth tergite.

Male terminalia: Epandrium completely divided middorsally, with 11-12 setae near posterior to ventral margins (Fig. 31). Surstylus slightly pubescent only on basal mesal surface, not expanded at posteroventral corner, with four long prensisetae on distal margin (Fig. 31). Tenth sternite bilobed, laterally narrowly fused to surstyli (Fig. 31). Anterior portion of hypandrium entirely thin. Parameres lobe-shaped, with four sensilla medially (Fig. 33). Basiphallus divided into a pair of simple rods, each fused to base of paramere (Fig. 33).
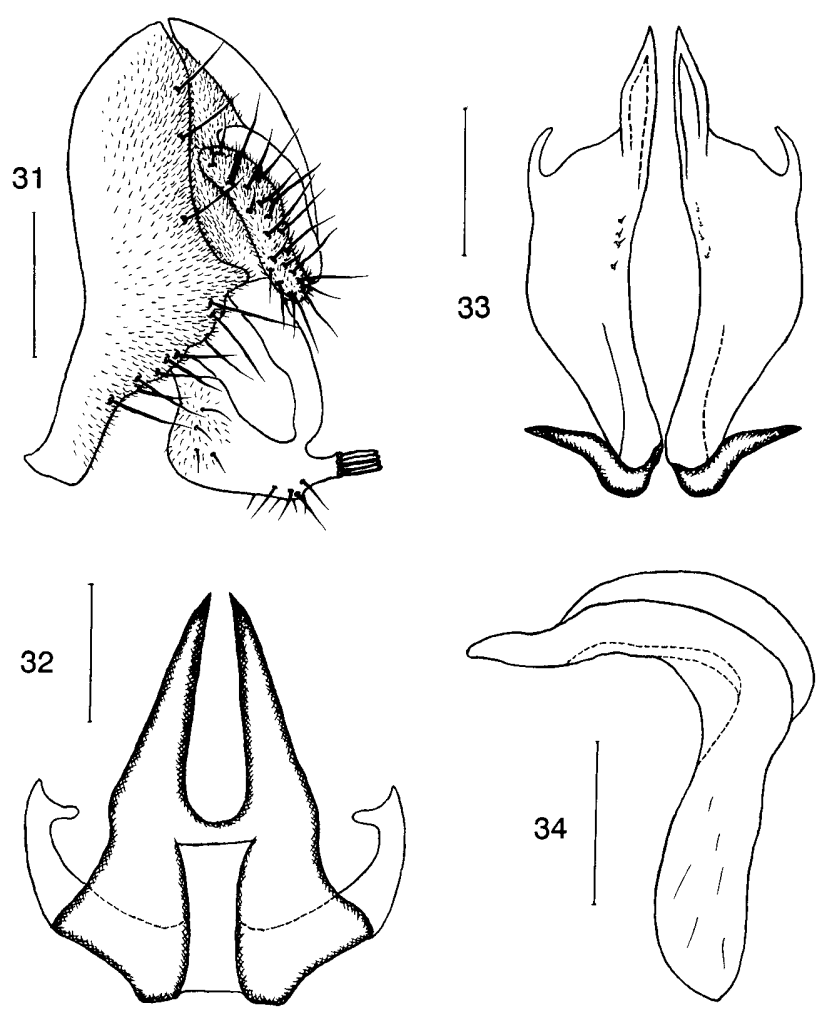

Figs 31-34. Amiota biacuta sp. n. $\mathbf{0}: 31$ - epandrium, cercus, surstylus and tenth sternite; 32 - gonopod (ventral view); 33 parameres and basiphallus (ventral view); 34 - aedeagal apodeme (ventral view). Scale $0.1 \mathrm{~mm}$.

Measurements. $\mathrm{BL}=2.78 \mathrm{~mm}$ in holotype; $\mathrm{ThL}=1.16 \mathrm{~mm}$; $\mathrm{WL}=2.30 \mathrm{~mm} ; \mathrm{WW}=1.13 \mathrm{~mm}$. Indices: $\mathrm{arb}=4 / 3$, avd $=0.55$, adf $=1.55, \mathrm{flw}=1.80, \mathrm{FW} / \mathrm{HW}=0.35, \mathrm{ch} / \mathrm{o}=0.06$, prorb $=$ $1.00, \mathrm{rcorb}=0.70, \mathrm{vb}=0.35, \mathrm{dcl}=0.55$, presctl $=0.60, \mathrm{sctl}=$ 1.15 , sterno $=0.85$, orbito $=1.80, \mathrm{dcp}=0.23, \mathrm{sctlp}=1.10, \mathrm{C}=$ $1.94,4 \mathrm{c}=1.50,4 \mathrm{v}=2.55,5 \mathrm{x}=1.10, \mathrm{ac}=3.87, \mathrm{M}=0.63, \mathrm{C} 3 \mathrm{~F}$ $=0.60$.

Type material. Holotype: $\widehat{\delta}$, Yunnan: Bamboo Temple, Kunming, alt. 2100 m, 21.vii.2004, around human eyes, H.-W. Chen (SCAU).

Etymology. A combination of the Latin words: bi- + acutus meaning two points, referring to the vertical lobe of gonopod with two heavily sclerotized, pointed processes.

Distribution. China (Yunnan: Kunming).

Remarks. This species is similar to $A$. paraspinata in the shape of paramere and basiphallus, but can be easily separated from it by the vertical lobe of gonopod (in $A$. paraspinata: vertical lobe of gonopod nearly squareshaped, medially slightly broadened).

\section{Amiota cultella sp. n. (Figs 35-38)}

Diagnosis. Vertical lobe of gonopod strongly sclerotized, acute, process apicomedially (Fig. 36); basiphallus slightly sclerotized (Fig. 37).

Description. Male: Hindleg femur with eight long setae posteroventrally; hindleg tibia with six setae ventrally.

Abdomen: Sixth tergite dorsally broadened, laterally tapering, reaching lateral margin of fifth tergite.

Male terminalia: Epandrium completely divided middorsally, with about 14 setae near posterior to ventral 


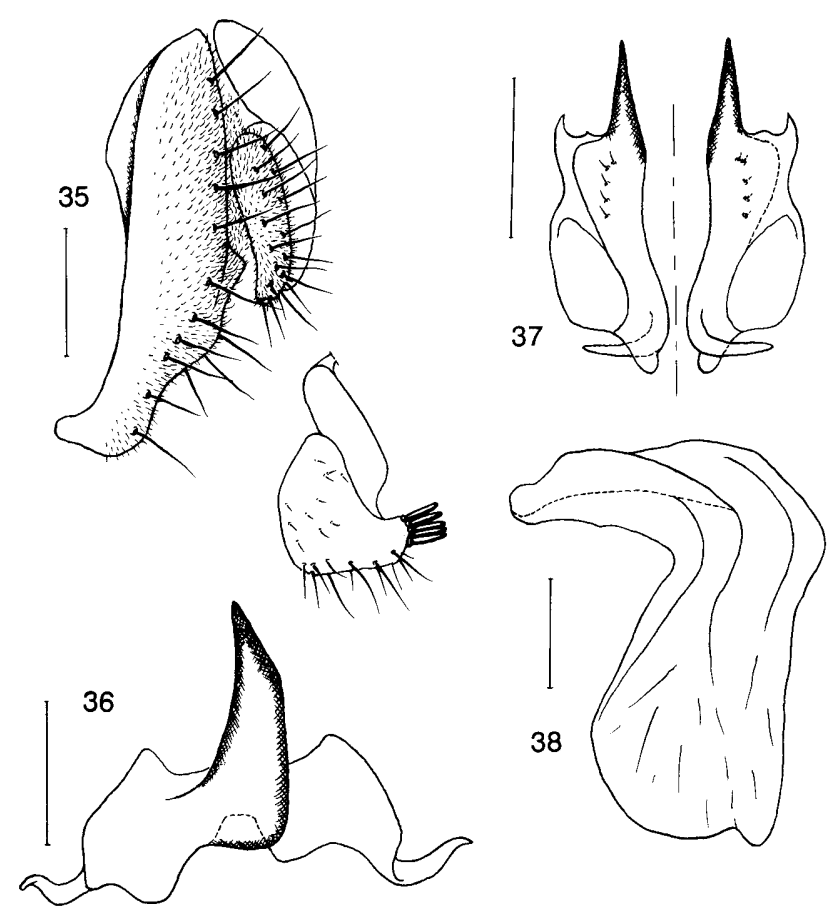

Figs 35-38. Amiota cultella sp. n. ठ̊: 35 - epandrium, cercus, surstylus and tenth sternite; 36 - gonopod (ventral view); 37 parameres and basiphallus (ventral view); 38 - aedeagal apodeme (lateroventral view). Scale $0.1 \mathrm{~mm}$.

margins (Fig. 35). Surstylus lacking pubescence, not expanded at posteroventral corner, with five long prensisetae on distal margin (Fig. 35). Tenth sternite bilobed, but not divided medially, laterally narrowly fused to surstyli (Fig. 35). Anterior portion of hypandrium entirely thin. Paramere lobe-shaped, with four sensilla medially (Fig. 37). Basiphallus divided into a pair of simple rods, each fused to base of paramere (Fig. 37).

Measurements. $\mathrm{BL}=2.74 \mathrm{~mm}$; $\mathrm{ThL}=1.06 \mathrm{~mm} ; \mathrm{WL}=2.45$ $\mathrm{mm} ; \mathrm{WW}=1.07 \mathrm{~mm}$. Indices: $\mathrm{arb}=3 / 3$, avd $=0.50$, adf $=1.50$, $\mathrm{flw}=1.30, \mathrm{FW} / \mathrm{HW}=0.35, \mathrm{ch} / \mathrm{o}=0.07$, prorb $=1.05$, rcorb $=$ $0.55, \mathrm{vb}=0.40, \mathrm{dcl}=0.60$, presctl $=0.70, \mathrm{sctl}=1.10$, sterno $=$ 0.90, orbito $=2.00, \mathrm{dcp}=0.23$, sctlp $=1.10, \mathrm{C}=1.91,4 \mathrm{c}=$ $1.68,4 \mathrm{v}=2.73,5 \mathrm{x}=1.22, \mathrm{ac}=3.74, \mathrm{M}=0.65, \mathrm{C} 3 \mathrm{~F}=0.61$.

Type material. Holotype: $\delta$, Yunnan: Samage, Weixi, alt. 1900 m, 27.vii.2004, ex tree trunks, H.-W. Chen (SCAU).

Etymology. From the Latin word cultellus meaning knife, referring to the vertical lobe of gonopod with knife-like dorsadoriented process.

Distribution. China (Yunnan: Weixi).

Remarks. This species is similar to $A$. cuii in the vertical lobe of gonopod with dorsad-oriented process, but clearly distinguishable by the shape of this structure (in $A$. cuii: very narrow).

Amiota deltoidea sp. n. (Figs 39-41)

Diagnosis: Vertical lobe of gonopod nearly triangular, pointed apically (Fig. 40).

Description. Male: Hindleg femur with seven long setae posteroventrally; hindleg tibiae with about six setae ventrally.
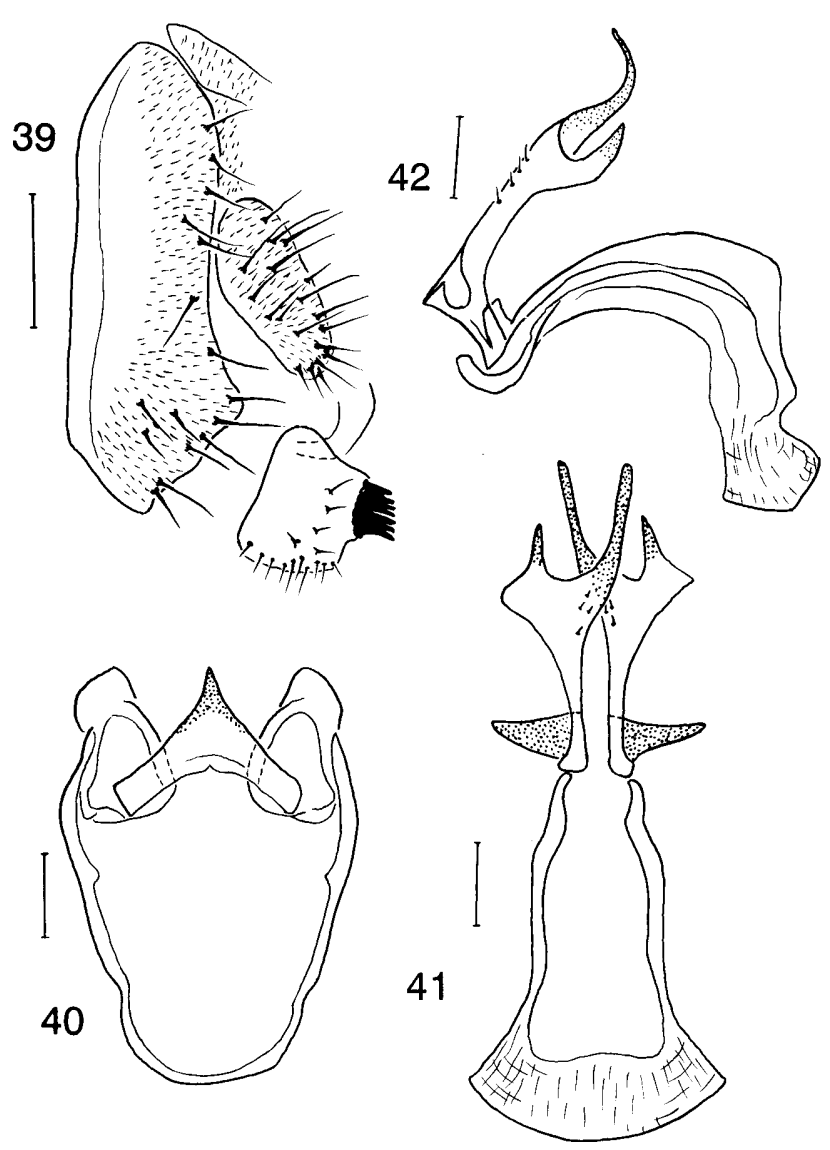

Figs 39-41. Amiota deltoidea sp. n. ô: 39 - epandrium, cercus and surstylus; 40 - gonopod and hypandrium (ventral view); 41 - parameres, basiphallus and aedeagal apodeme (ventral view); 42 - ditto (lateral view). Scale $0.1 \mathrm{~mm}$.

Abdomen: Sixth tergite dorsally broadened, laterally tapering, reaching lateral margin of fifth tergite.

Male terminalia: Epandrium completely divided middorsally, with $15-16$ setae near posterior to ventral margins (Fig. 39). Surstylus lacking pubescence, not expanded at posteroventral corner, with seven prensisetae on distal margin (Fig. 39). Anterior portion of hypandrium entirely thin (Fig. 40). Tenth sternite bilobed, laterally narrowly fused to surstyli. Paramere nearly lobeshaped, with four sensilla medially (Figs 41, 42). Basiphallus divided into a pair of simple rods, each fused to base of paramere (Fig. 41).

Measurements. $\mathrm{BL}=3.16 \mathrm{~mm}$ in holotype (range in 4 paratypes: $3.10-3.25), \mathrm{ThL}=1.53 \mathrm{~mm}(1.45-1.60), \mathrm{WL}=3.18 \mathrm{~mm}$ (3.05-3.30), $\mathrm{WW}=1.30 \mathrm{~mm}(1.25-1.35)$. Indices: $a r b=4 / 4$ $(4 / 3-4)$, avd $=0.50(0.50)$, adf $=1.00(1.00)$, flw $=1.35$ $(1.35-1.45), \mathrm{FW} / \mathrm{HW}=0.35(0.35), \mathrm{ch} / \mathrm{o}=0.10(0.10)$, prorb $=$ $1.00(1.00-1.10)$, rcorb $=0.65(0.55-0.65), \mathrm{dcl}=0.58$ $(0.50-0.60)$, prsctl $=0.62(0.65-0.70)$, sctl $=1.15(1.10-1.15)$, sterno $=$ ?, orbito $=1.77(1.67-1.86), \mathrm{dcp}=0.30(0.27-0.33)$, sctlp $=1.09(1.00-1.17), \mathrm{C}=2.14(2.06-2.21), 4 \mathrm{c}=1.83$ (1.65-2.00), $4 \mathrm{v}=3.41(3.05-3.67), 5 \mathrm{x}=1.35(1.25-1.45), \mathrm{ac}=$ $4.33(4.13-4.53), \mathrm{M}=0.85(0.75-0.94), \mathrm{C} 3 \mathrm{~F}=0.23(0.22-$ $0.24)$.

Type material. Holotype: $\delta$, Yunnan: Lake Bitahai, Shangrila, alt. $3000 \mathrm{~m}$, 14.vii.1993, around human eyes, W. Wang (KIZ). Paratypes: $1 \hat{0}$, same data as holotype (KIZ); Yunnan: 


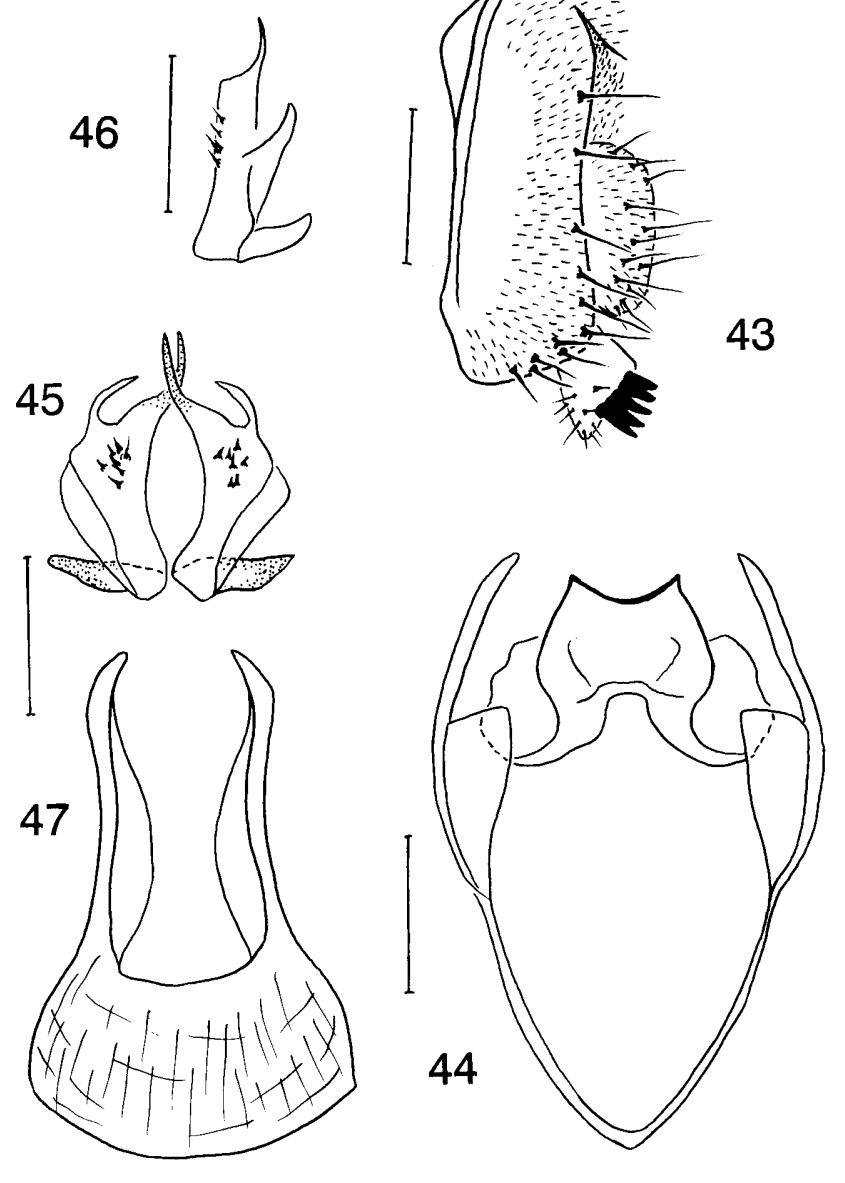

Figs 43-47. Amiota pianmensis sp. n. ồ: 43 - epandrium, cercus and surstylus; 44 - gonopod and hypandrium (ventral view); 45 - parameres and basiphallus (ventral view); 46 - ditto (lateral view); 47 - aedeagal apodeme (ventral view). Scale 0.1 $\mathrm{mm}$.

4ठิ, Tuguanchun, Shangrila, 4.vi.1994, around human eyes, W.-X. Zhang (20, PKU; 20 , SCAU).

Etymology. A combination of the Greek words: delta and -oideos meaning triangular, referring to the triangular vertical lobe of gonopod.

Distribution. China (Yunnan: Shangrila).

Remarks. This species is similar to A. watabei in the shape of the vertical lobe of gonopod, but they are clearly distinguishable by the surstylus and the paramere (in $A$. watabei: surstylus with five prensisetae on distal margin; paramere without lateral process).

\section{Amiota pianmensis sp. n. (Figs 43-47)}

Diagnosis. Epandrium constricted mid-dorsally more deeply than one-half of width (Fig. 43); vertical lobe of gonopod slightly concave apicomedially (Fig. 44).

Description. Male: Hindleg femur with about seven long setae posteroventrally; hindleg tibiae with about eight setae ventrally.

Abdomen: Sixth tergite dorsally broadened, laterally tapering, reaching lateral margin of fifth tergite.

Male terminalia: Epandrium with 15-16 setae near posterior to ventral margins (Fig. 43). Surstylus lacking pubescence, slightly expanded at posteroventral corner, with five short prensisetae on distal margin (Fig. 43). Tenth sternite bilobed, but not divided medially, laterally narrowly fused to surstyli. Anterior portion of hypandrium entirely thin (Fig. 44). Paramere lobe-shaped, with about seven sensilla medially (Fig. 45). Basiphallus divided into a pair of simple rods, each fused to base of paramere (Figs 45, 46).

Measurements. $\mathrm{BL}=2.35 \mathrm{~mm}$ in holotype, $\mathrm{ThL}=1.23 \mathrm{~mm}$, $\mathrm{WL}=2.10 \mathrm{~mm}, \mathrm{WW}=1.10 \mathrm{~mm}$. Indices: $\mathrm{arb}=4 / 3$, avd $=0.65$, adf $=1.15, \mathrm{flw}=1.55, \mathrm{FW} / \mathrm{HW}=0.42, \mathrm{ch} / \mathrm{o}=0.10$, prorb $=$ 1.05, rcorb $=0.68, \mathrm{dcl}=0.43$, presctl $=0.61, \mathrm{sctl}=1.10$, sterno $=0.79$, orbito $=1.67, \mathrm{dcp}=0.28$, sctlp $=1.00, \mathrm{C}=1.89,4 \mathrm{c}=$ $1.87,4 \mathrm{v}=3.30,5 \mathrm{x}=1.04, \mathrm{ac}=5.09, \mathrm{M}=0.87, \mathrm{C} 3 \mathrm{~F}=0.68$.

Type material. Holotype: $\hat{o}$, Yunnan: Changyanhe, Pianma, Lushui, 28.iv.1993, around human eyes, L.-X. Han (KIZ). Paratype: $\hat{\delta}$, same data as holotype except alt. $1500 \mathrm{~m}, 15$.viii.2000, H.-W. Chen (SCAU).

Etymology. In reference to the type locality.

Distribution. China (Yunnan: Lushui).

Remarks. This species is related to A. jizushanensis, but they are clearly distinguishable by the vertical lobe of gonopod (in A. jizushanensis: vertical lobe of gonopod with two thin processes apicolaterally).

\section{Amiota setosa sp. n. (Figs 48-51)}

Diagnosis. All femora, mid- and hindleg tibiae black except yellow knees; paramere and basiphallus fused basomedially: paramere distally bifurcated (Fig. 51); basiphallus apically curved hook-like (Fig. 51).

Description. Male: Acrostichal setulae in about 14-16 irregular rows. Foreleg tibia and all tarsomeres yellow; hindleg femur basoventrally slightly extended, distally with a row of long anteroventral setae; hindleg tibia apicodorsally slightly extended, ventrally lacking longer setae.

Abdomen: Lateral margin of sixth tergite slightly narrower than dorsomedian part.

Male terminalia: Epandrium dorso-medially entirely divided into two lateral lobes, with very dense setae (Fig. 48). Surstylus lacking pubescence, with finger-like process at posteroventral corner, about 13 long prensisetae on distal margin (Fig. 48). Anterior portion of hypandrium broadened laterally (Fig. 49). Vertical lobe of gonopod round, weakly sclerotized (Fig. 49).

Measurements. $\mathrm{BL}=4.60 \mathrm{~mm}$ in holotype (range in 4 paratypes: $4.40-4.80), \mathrm{ThL}=2.24 \mathrm{~mm}(2.18-2.30), \mathrm{WL}=3.73 \mathrm{~mm}$ $(3.60-3.85), \mathrm{WW}=1.69 \mathrm{~mm}(1.68-1.70)$. Indices: $\mathrm{arb}=$ $5-6 / 4-5(5-6 / 4-5)$, avd $=0.70(0.60-0.70)$, adf $=1.20$ $(1.20-1.30), \quad$ flw $=2.00(2.00-2.20), \quad \mathrm{FW} / \mathrm{HW}=0.42$ $(0.40-0.43), \mathrm{ch} / \mathrm{o}=0.10(0.08-0.11)$, prorb $=1.04(1.03-1.04)$, rcorb $=0.83(0.77-0.88), \mathrm{dcl}=0.50(0.50-0.55)$, presctl $=0.63$ $(0.60-0.70)$, sctl $=1.05(1.00-1.10)$, sterno $=0.88(0.85-0.91)$, orbito $=1.73(1.67-1.78), \mathrm{dcp}=0.27(0.26-0.29)$, sctlp $=1.12$ (1.08-1.18), C $=2.45(2.36-2.54), 4 \mathrm{c}=1.25(1.20-1.30), 4 \mathrm{v}=$ $2.58(2.37-2.78), 5 \mathrm{x}=0.97(0.94-1.00), \mathrm{ac}=3.32(3.04-3.60)$, $\mathrm{M}=0.52(0.47-0.56), \mathrm{C} 3 \mathrm{~F}=0.85(0.81-0.88)$.

Type material. Holotype: $\widehat{\delta}$, Yunnan: Pengdang, Gongshan, alt. $1475 \mathrm{~m}, 23 . v i .1993$, around human eyes, W.-X. Zhang (KIZ). Paratypes: 10, same data as holotype; Yunnan: $3 \hat{0}$, Tuguanchun, Shangrila, alt. 2500 m, 3.vi.1994, around human 
eyes, W.-X. Zhang (PKU); 30, Samage, Weixi, alt. $1900 \mathrm{~m}$, 27.vii.2004, ex tree trunks, H.-W. Chen (SCAU).

Etymology. From the Latin word setosus meaning with many setae, referring to the epandrium with dense setae.

Distribution. China (Yunnan: Gongshan, Shangrila, Weixi).

Remarks. This species is similar to A. albilabris in the legs color and the vertical lobe of gonopod, but they can be distinguished by the paramere and the basiphallus (in A. albilabris: paramere and basiphallus separated from each other; paramere slender, distally not bifurcated).

\section{Amiota rufescens species-group}

Amiota (Amiota) rufescens species-group, Chen \& Toda, 2001: 1547; Chen et al., 2004: 65; Chen et al., 2005: 300.

Diagnosis. Prensisetae on surstylus long, pointed apically.

Included species. A. leucostoma Loew, 1862; A. magniflava Chen \& Toda, 2001; A. rufescens (Oldenberg, 1914); A. stylopyga Wakahama \& Okada, 1958.

\section{Amiota magniflava Chen \& Toda, 2001}

Amiota (Amiota) magniflava Chen \& Toda, 2001: 1547.

Specimen examined. Yunnan: 4$\hat{\jmath}$, Samage, Weixi, 27.-30. vii.2004, alt. $1900 \mathrm{~m}$, ex tree trunks, Y.-F. Li and H.-W. Chen.

Distribution. China (Hubei, Yunnan: Weixi, Jingdong).

Amiota stylopyga Wakahama \& Okada, 1958

Amiota (Amiota) stylopyga Wakahama \& Okada, 1958: 109.

Specimens examined. Yunnan: 1 $\hat{0}$, Samage, Weixi, 27.vii.2004, alt. 1900 m, ex tree trunks, H.-W. Chen.

Distribution. Russia (Irkutsk and Buryatiya Regions, Amur Region, Khabarovsk Region, Ussuri Region, Sakhalin), China (Heilongjiang, Jilin, Liaoning, Yunnan*: Weixi), Korea, Japan (Hokkaido, Honshu).

\section{Ungrouped species}

\section{Amiota acuta Okada, 1968}

Amiota (Amiota) acuta Okada, 1968: 306; Chen \& Toda, 2001: 1549.

Specimen examined. Yunnan: $3 \hat{\sigma}$, Bamboo Temple, Kunming, alt. $2100 \mathrm{~m}, 21 . v i i .2004$, around human eyes, H.W. Chen.

Distribution. China (Yunnan: Binchuan, Kunming), Japan (Honshu)

\section{Amiota dentata Okada, 1971}

Amiota (Amiota) dentata Okada, 1971: 87; Máca \& Lin, 1993: 2; Chen \& Toda, 2001: 1550.

Specimens examined. Yunnan: $3 \hat{\sigma}$, Bamboo Temple, Kunming, alt. $2100 \mathrm{~m}, 21$.vii.2004, around human eyes, H.W. Chen.

Distribution. China (Liaoning, Taiwan, Hubei, Yunnan: Kunming, Binchuan, Ningliang), Japan (Hokkaido, Honshu).

\section{Amiota furcata Okada, 1971}

Amiota (Amiota) furcata Okada, 1971: 85 [Amiota (Amiota) alboguttata, forma furcata Okada, 1960: 96 (part.)]; Máca \& Lin, 1993: 2; Chen \& Toda, 2001: 1550.

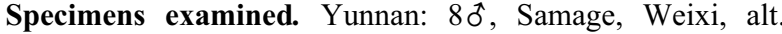
$1900 \mathrm{~m}, 27 .-30 . v i i .2004,3 \hat{0}$ around human eyes, $5 \hat{0}$ ex tree trunks, Y.-F. Li and H.-W. Chen; 1 $\widehat{\sigma}$, Benzilan, Deqin, alt 2700 m, 31.vii.2004, ex tree trunks, Y.-F. Li.

Distribution. China (Taiwan, Hubei, Hunan, Fujian, Sichuan, Yunnan: Kunming, Binchuan, Ninglang, Weixi, Deqin), Japan (Hokkaido, Honshu, Kyushu).
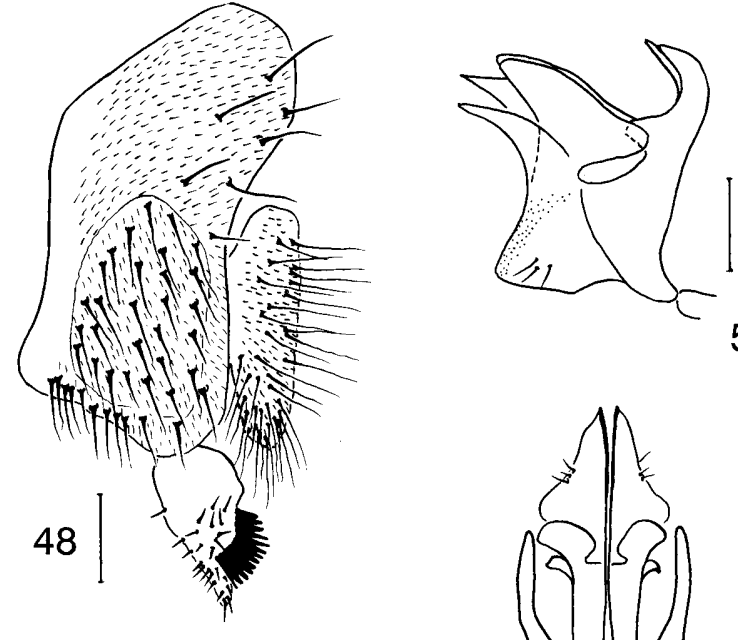

51
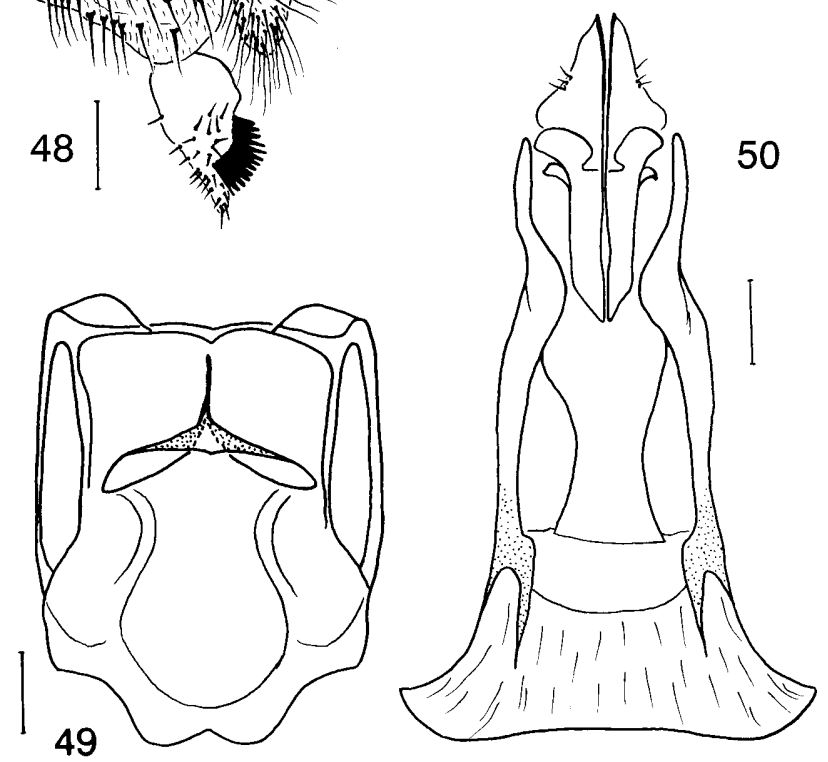

Figs 48-51. Amiota setosa sp. n. đ: 48 - epandrium, cercus and surstylus; 49 - gonopod and hypandrium (ventral view); 50 - parameres, basiphallus and aedeagal apodeme (ventral view); 51 - parameres and basiphallus (lateral view). Scale $0.1 \mathrm{~mm}$.

\section{Amiota fuscata Chen \& Zhang, 2005}

Amiota (Amiota) fuscata Chen \& Zhang in Chen et al., 2005: 301.

Specimens examined. Holotype: $\widehat{\delta}$, Yunnan: Gaoligongshan, Pianma, Lushui, 15.viii.2000, ex tree trunk, H.-W. Chen (SCAU).

Distribution. China (Yunnan: Lushui, Jingdong).

\section{Amiota subfurcata Okada, 1971}

Amiota (Amiota) subfurcata Okada, 1971: 85 [Amiota (Amiota) alboguttata, forma furcata Okada, 1960: 96 (part.)]; Máca \& Lin, 1993: 2; Chen \& Toda, 2001: 1551.

Amiota (Amiota) pacifica Sidorenko, 1989: 63 (synonymized by Sidorenko, 1992: 260).

Specimens examined. Yunnan: 24ð, Samage, Weixi, 27.-30. vii.2004, $13 \delta$ around human eyes, $11 \delta^{\Uparrow}$ ex tree trunks, Y.-F. Li and H.-W. Chen.

Distribution. Russia (East Siberia, Far East), China (Jilin, Liaoning, Beijing, Zhejiang, Fujian, Taiwan, Guangdong, Guangxi, Sichuan, Yunnan: Kunming, Binchuan, Weixi), Japan (Hokkaido, Honshu, Kyushu).

Amiota bifoliolata sp. n. (Figs 52-55)

Diagnosis. Gonopods close to each other, anteriorly forming nearly triangular, vertical lobe, sclerotized only 

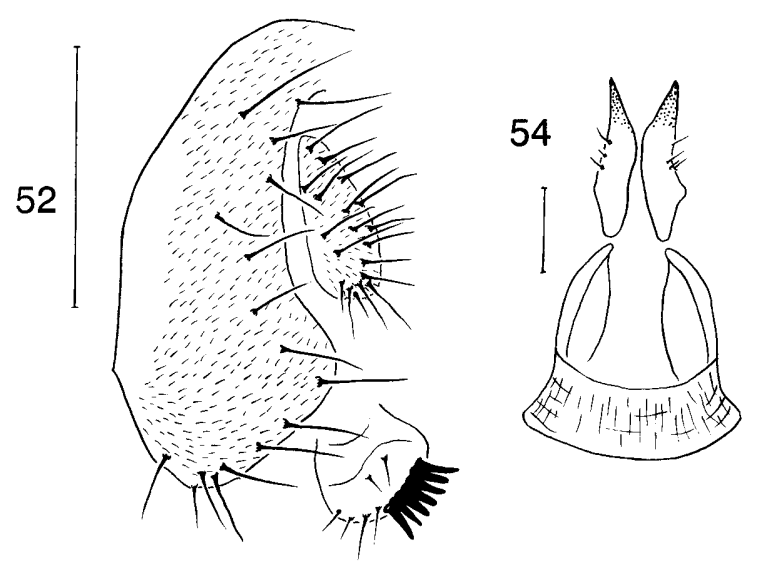

55

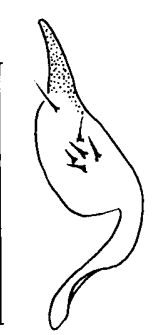

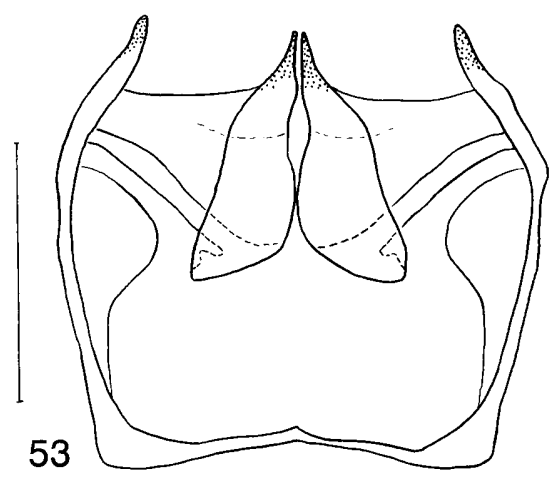

Figs 52-55. Amiota bifoliolata sp. n. ỗ: 52 - epandrium, cercus and surstylus; 53 - gonopod and hypandrium (ventral view); 54 - parameres and aedeagal apodeme (ventral view); 55 - paramere (lateral view). Scale $0.1 \mathrm{~mm}$.

apically (Fig. 53); paramere submedially strongly curved in lateral view (Fig. 55).

Description. Male: Hindleg femur and tibia lacking special setae.

Abdomen: Lateral margin of sixth tergite slightly narrower than dorsomedian part.

Male terminalia: Epandrium not constricted middorsally, with about 15 setae near posterior to ventral margins (Fig. 52). Surstylus lacking pubescence, with seven long prensisetae on distal margin (Fig. 52). Anterior portion of hypandrium thin at middle (Fig. 53). Paramere lobe-shaped, weakly sclerotized, basally slender in lateral view, medially expanded and with about six sensilla, apically pointed (Figs 54, 55). Basiphallus lost or indistinguishably fused to paramere (Figs 54, 55).

Measurements. $\mathrm{BL}=1.95 \mathrm{~mm}$, $\mathrm{ThL}=1.08 \mathrm{~mm}, \mathrm{WL}=1.50$ $\mathrm{mm}, \mathrm{WW}=1.00 \mathrm{~mm}$. Indices: $\mathrm{arb}=5 / 4$, avd $=0.50$, adf $=1.10$, $\mathrm{flw}=1.30, \mathrm{FW} / \mathrm{HW}=0.44, \mathrm{ch} / \mathrm{o}=0.09$, prorb $=1.00$, rcorb $=$ $0.80, \mathrm{vb}=0.40, \mathrm{dcl}=0.53$, presctl $=0.37, \mathrm{sctl}=0.90$, sterno $=$ 0.93 , orbito $=2.00, \mathrm{dcp}=0.35, \mathrm{sctlp}=1.25, \mathrm{C}=1.40,4 \mathrm{c}=$ $2.15,4 \mathrm{v}=3.10,5 \mathrm{x}=1.73, \mathrm{ac}=4.78, \mathrm{M}=0.95, \mathrm{C} 3 \mathrm{~F}=0.67$.

Type material. Holotype: $\delta$, Yunnan: Pula River, Gongshan, 26.vi.1994, around human eyes, W.-X. Zhang (KIZ).

Etymology. A combination of the Latin words: bi- and foliolatus meaning two leaves, referring to the leaf-like parameres.

Distribution. China (Yunnan: Gongshan).
Remarks. This species is similar to A. kamui Chen \& Toda, 2001 in the shape of paramere and the medially separated gonopods, but can be clearly distinguished by the epandrium and the gonopods (in A. kamui: epandrium constricted more than one-half width mid-dorsally; gonopods heavily sclerotized).

Key to 39 species of the genus Amiota from Hengduan Mountains - males

1 Surstylus with a few aristate processes on mesal surface; vertical lobe of gonopod with two sclerotized, basally fused M-shaped processes (basdeni species-group). ....... 2

- Surstylus without aristate processes; vertical lobe of gonopod without M-shaped process. . . . . . . . . 12

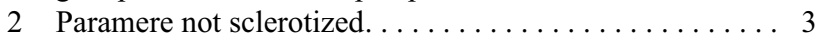

Paramere sclerotized. .................... 5

3 Basiphallus slender, rod-shaped; paramere nearly entirely pubescent. .................... A. yifengi sp. n.

- Basiphallus short, nearly triangular; paramere glabrous or pubescent on small part. ................. 4

4 Paramere lobe-shaped, submedially pubescent.......... .................. brevipartita Chen \& Gao

Paramere rod-shaped, glabrous. . ............... .................. angulisternita Chen \& Liu

5 Basiphallus strongly sclerotized, black. ......... 6

Basiphallus sclerotized, not black. ............ 8

6 Paramere curved apically; basiphallus very small, entirely fused to base of paramere....... A. onchopyga Nishiharu

- Paramere gently curved in its entire length; basiphallus only slightly shorter than paramere. ............ 7

7 Basiphallus basally fused to paramere; parameres divergent. . ..................... A. curvispina Chen \& Gao

- Basiphallus basally articulated with paramere; parameres close to each other......... A. longispinata Chen \& Gao

8 Paramere distally tripartite, basally fused to basiphallus.... . ...................... A. multispinata sp. n. Paramere distally not tripartite, basally articulated with basi-

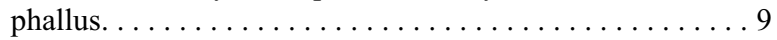

9 Paramere apically pointed........... gracilenta sp. n.

- Paramere apically blunt or round. .............. 10

10 Paramere basally curved in lateral view.............. $\ldots \ldots \ldots \ldots \ldots \ldots \ldots$ A. lipingae Chen \& Gao

Paramere nearly straight. ................. 11

11 Basiphallus medially broadened in lateral view and with one small triangular projection; paramere thick, finely serrated on apical margin.................... A. gaoi sp. n.

- Basiphallus slender, medially without projection; paramere lacking fine serrations on apical margin, narrowed from distal one-third in lateral view. . . . . A. macai Chen \& Toda

12 Hindleg femur ventro-basally with nearly hyaline, small, lobe-like flap (taurusata species-group). . . . . . . . 13

- Hindleg femur sometimes basally dilated, but without lobelike flap. ...................... 15

13 All femora dark brown; hindleg femur curved basally; vertical lobe of gonopod nearly triangular. . . . . . . . . . . . ................... femorata Chen \& Takamori

- Legs entirely yellow; hindleg femur straight; vertical lobe of gonopod nearly round. . . . . . . . . . . . . . 14

14 Parameres and basiphallus asymmetrical; basiphallus not bifurcated. . . . . . . . A. asymmetrica Chen \& Takamori Parameres and basiphallus nearly symmetric; basiphallus deeply bifurcated A. aquilotaurusata Takada, Beppu \& Toda 
15 Hindleg tibia ventrally with a row of long setae; hindleg second to fifth tarsomeres broadened: second tarsomere at least shorter than half its width (alboguttata species-group). . .................................. 16

Hindleg tibia ventrally without long setae; hindleg second to fifth tarsomeres not broadened: second tarsomere longer than half its width. . . . . . . . . . . . . . . . 32

16 All femora, mid- and hindleg tibiae black except for yellow joints. ........................ setosa sp. n. Legs entirely yellow. . . . . . . . . . . . . . . . 17

17 Basiphallus divided into a pair of deeply bifurcated processes. . . . . . . . . . . . . . . . . A. trifurcata Okada Basiphallus divided into a pair of simple rods........ 18

18 Vertical lobe of gonopod slightly concave apicomedially... ........................ Aianmensis sp. $\mathrm{n}$. Vertical lobe of gonopod apicomedially with distinct process(es) . . . . . . . . . . . . . . . . . . . . . . 19

19 Basiphallus slightly sclerotized, not black. . . . . . . . 20 Basiphallus strongly sclerotized, black. ......... 21

20 Vertical lobe of gonopod strongly sclerotized, apicomedially with one long, acute process........... A. cultella sp. n.

- Vertical lobe of gonopod weakly sclerotized, apicomedially with two small processes. . A. jizushanensis Chen \& Watabe

21 Vertical lobe of gonopod medially divided into lateral lobes. ................... dehiscentia Chen \& Watabe Vertical lobe of gonopod medially not divided. . . . . . 22

22 Vertical lobe of gonopod apically with three processes..... ..................... nozawai Chen \& Watabe Vertical lobe of gonopod apically with one or two processes. ................................ 23

23 Vertical lobe of gonopod apically with one process.... 24 Vertical lobe of gonopod apically with two processes.... 31

24 Vertical lobe of gonopod long, rod-shaped, with one acute projection posteromedially............. A. bacillia $\mathrm{sp} . \mathrm{n}$. Vertical lobe of gonopod broad and large, without acute projection posteromedially................. 25

25 Vertical lobe of gonopod nearly triangular. . . . . . . . . . . Vertical lobe of gonopod basally roundish or quadrate. . . 26

26 Vertical lobe of gonopod basally quadrate. . . . . . . 27 Vertical lobe of gonopod basally roundish. . . . . . . 29

27 Vertical lobe of gonopod strongly sclerotized, entirely black. A. latitabula Chen \& Watabe

- Vertical lobe of gonopod only apically and laterally black . . ............................... 28

28 Vertical lobe of gonopod acute apicolaterally, clearly broader than its height. ...... A. shangrila Chen \& Watabe

- Vertical lobe of gonopod round apicolaterally, its height equal with maximum. ............ A. angustifolia $\mathrm{sp.}$.

29 Apical process of vertical lobe of gonopod thick, rod-shaped, apically round in ventral view. ........... ................. A. ailaoshanensis Chen \& Watabe

- Apical process of vertical lobe of gonopod nearly triangular, apically pointed in ventral view. . . . . . . . . . . 30

30 Vertical lobe of gonopod mostly sclerotized, as high as wide. .................... A. arcuata Chen \& Watabe - Vertical lobe of gonopod sclerotized only apically, higher than wide. . . . . ....... A. luguhuensis Chen \& Watabe

31 Vertical lobe of gonopod distinctly higher than wide....... ............................ biacuta $\mathrm{sp.}$. .

Vertical lobe of gonopod as high as wide. ... . . . paraspinata Chen \& Watabe

32 Body large (more than $4.0 \mathrm{~mm}$ in length); prensisetae on surstylus long, apically pointed (rufescens species-group)...
- Body small (less than $4.0 \mathrm{~mm}$ in length); prensisetae on surstylus short and apically blunt (ungrouped species). . . 34

33 Paramere lobe-like, not black; vertical lobe of gonopod short. . . . . . .......... magniflava Chen \& Toda

- Paramere slender, rod-like, black, heavily sclerotized; vertical lobe of gonopod as long as paramere............. .................. A. stylopyga Wakahama \& Okada

34 All femora dark brown; paramere and basiphallus entirely separated from each other. ...... A. fuscata Chen \& Zhang All femora yellow; basiphallus fused to parameres. . . . . 35

35 Fifth sternite triangularly notched on posterior margin, with dense, long setae. . . . . . . . . . . . . . 36

- Fifth sternite quadrate, not notched postero-medially, without dense, long setae. . . . . . . . . . . . . 37

36 Surstylus with 11-12 prensisetae; midleg tibia medioventrally with $5-7$ erected setae longer than others......... . . . . . . . . . . . . . . . . . . . . . A. furcata Okada

- Surstylus with 6-7 prensisetae; midleg tibia ventrally without any long, erected seta. ....... A. subfurcata Okada

37 Vertical lobe of gonopod narrowly elongated apically, not divided medially................. A. acuta Okada - Vertical lobe of gonopod medially divided. . . . . . . . 38

38 Epandrium entirely divided mid-dorsally; vertical lobe of gonopod with large sclerotized serrations apically and laterally. ....................... A. dentata Okada

- Epandrium slightly constricted mid-dorsally; vertical lobe of gonopod apically pointed. .......... bifoliolata $\mathrm{sp} . \mathrm{n}$.

ACKNOWLEDGEMENTS. We would like to express our sincere thanks to H. Shima (Graduate School of Social and Cultural Studies, Kyushu University, Japan), W. Wang (KIZ), L.-X. Han (KIZ) J.-J. Gao (Yunnan University, China) and Y.-F. Li (SCAU), who helped us in field collections and provided us with invaluable specimens. This work was supported by the Scientific Research Foundation for the Returned Overseas Chinese Scholars, State Education Ministry of China; National Natural Science Foundation of China (Nos 30270186, 30470212) and Natural Science Foundation of SCAU (No. K03209); International Scientific Research Program (Field Research) of Ministry of Education, Culture, Sports, Science and Technology, Japan (No. 05041099).

\section{REFERENCES}

Chen H.-W. \& Aotsuka T. 2003: A survey of the genus Leucophenga (Diptera, Drosophilidae) in Iriomote-jima of Japan, with descriptions of three new species. Can. Entomol. 135: $1-16$.

Chen H.-W. \& Toda M.J. 1998a: Amiota (Amiota) apodemata species-group, with descriptions of two new species from Southeast Asia (Diptera, Drosophilidae). Entomol. Sci. 1: 271-275.

Chen H.-W. \& Toda M.J. 1998b: Amiota (Amiota) sinuata species-group, with descriptions of five new species from Southeast Asia (Diptera, Drosophilidae). Entomol. Sci. 1: 409-416.

Chen H.-W. \& Toda M.J. 2001: A revision of the Asian and European species in the subgenus Amiota Loew (Diptera, Drosophilidae) and establishment of species-groups based on phylogenetic analysis. J. Nat. Hist. 35: 1571-1563.

Chen H.-W., Zhang C.-T. \& Liu G.-C. 2004: New species and new records of the subgenus Amiota s. str. Loew (Diptera, Drosophilidae) from North America, East Asia and Oceania. Ann. Soc. Entomol. Fr. 40: 59-67.

Chen H.-W., Watabe H., Gao J.-J., Takamori H., Zhang Y.-P. $\&$ AotsuKa T. 2005: Species diversity of the subgenus Amiota 
(s. str.) Loew, 1862 (Diptera, Drosophilidae) in southern China. J. Nat. Hist. 39: 265-310.

Loew H. 1862: Diptera Americae septentrionalis indigena. Centuria secunda. Berl. Entomol. Z. 6: 185-232.

MÁcA J. 1980: European species of the subgenus Amiota s. str. (Diptera, Drosophilidae). Acta Entomol. Bohemoslov. 77: 328-346.

MÁcA J. 2003: Taxonomic notes on the genera previously classified in the genus Amiota Loew (Diptera: Drosophilidae, Steganinae). Acta Univ. Carol. (Biol.) 47: 247-274.

MÁca J. \& Lin F. J. 1993: The Drosophilidae of Taiwan: Genera Amiota (excluding subgenus Phortica) and Leucophenga (Nankangomyia subg. nov.). Bull. Inst. Zool. Acad. Sin. 32 $1-11$.

MeYers N. 1988: Threatened biotas: "Hot spots" in tropical forests. Environmentalist 8: 187-208.

NishinaRu S. 1979: Three new species of Drosophilidae from Japan (Diptera). Kontyu (Tokyo) 47: 38-43.

OKadA T. 1960: On the Japanese species of the genus Amiota Loew (Diptera, Drosophilidae). Mushi 34: 89-102.

OKADA T. 1968: Addition to the fauna of the family Drosophilidae of Japan and adjacent countries (Diptera, Drosophilidae). I. Genera Stegana, Amiota, Leucophenga, and Microdrosophila, with discussion on the homology of phallic organs. Kontyu (Tokyo) 36: 303-323.

OKADA T. 1971: A revision and taxometric analysis of the genus Amiota Loew of Japan and adjacent countries (Diptera, Drosophilidae). Kontyu (Tokyo) 39: 82-98.
RaVen P.H. \& Axelrod D.I. 1978: Origin and Relationships of the California Flora. California Native Plant Society, University of California, $134 \mathrm{pp}$.

Sidorenko V.S. 1989: Drosophilid flies of the genus Amiota (Diptera, Drosophilidae) from Primorie Territory. Zool. J. 68: 60-66.

Sidorenko V.S. 1992: Family Drosophilidae. Insects of Khingansky Reserve. Part II. Dalnauka Publishing Huse, Vladivostok, pp. 259-264.

TAKada H., Beppu K. \& Toda M.J. 1979: Drosophila survey of Hokkaido, XXXVI. New and unrecorded species of Drosophilidae (Diptera). J. Fac. Gener. Educ. Sapporo Univ. 14: 105-129.

Toda M.J., Sidorenko V.S., Watabe H., Kholin S.K. \& VINOKUROV N.N. 1996: A revision of the Drosophilidae (Diptera) in East Siberia and Russian Far East: Taxonomy and biogeography. Zool. Sci. 13: 455-477.

WAKAHAMA K.I. \& OKADA T. 1958: Drosophila survey of Hokkaido VIII. Description of a new species of the genus Amiota (Drosophilidae) from Japan. Annot. Zool. Jap. 31: 109-112.

WheELER M.R. 1952: The Drosophilidae of the Nearctic region, exclusive of the genus Drosophila. Univ. Texas Publ. 5204: $162-218$.

WILsON E.O. 1992: The Diversity of Life. Belknap Press of Harvard University Press, WW Norton Company, New York, Cambridge, $424 \mathrm{pp}$.

Wu C.-Y. 1988: Hengduan Mountain flora and her significance. J. Jap. Bot. 63: 297-311.

Received March 31, 2005; revised and accepted November 15, 2005

\section{BOOK REVIEW}

Raman A., Schaefer C.W. \& Withers T.M. (eds): BIOLOGY ECOLOGY, AND EVOLUTION OF GALL-INDUCING ARTHROPODS. VOL. 1, 2. Science Publishers, Enfield (NH), USA \& Plymouth, UK, 2005, xxi +817 pp. ISBN 1-57808262-5 (Set), 1-57808-345-1 (Vol. 1), 1-57808-346-X (Vol. 2). Price GBP 81.40

This two-volume book presents in 26 chapters the results of the work of forty nine biologists from seventeen countries. It is devoted to interesting and remarkable groups of animals that cause galls on plants. It was edited by: Prof. Anantanarayanan Raman of the University of Sydney, Orange, New South Wales, Australia; Prof. Carl W. Schaefer of the University of Connecticut, Storrs, Connecticut, USA and the entomologist Toni M.
Withers of the New Zealand Crown Research Institute, Forest Research, Rotorua, New Zealand. Before the preface, there is a dedication by all co-authors of this book to the forty eight researchers from all over the world for their contribution to the study of gall-inducing arthropods.

This new book appeared twenty years after the publication of the book by T.N. Ananthakrishnan (1984) "Biology of Gall Insects" (Oxford \& IBH Publishing, New Delhi, Bombay, Calcutta, $362 \mathrm{pp}$.) in which fourteen authors summarized the then knowledge on gall-causing insects in eleven chapters. Ananthakrishnan's book is kept as the general starting point for summarizing the progress in cecidological studies. The present book brings an overview and new results obtained in the last twenty years, when new trails were discovered, hidden secrets of gall 